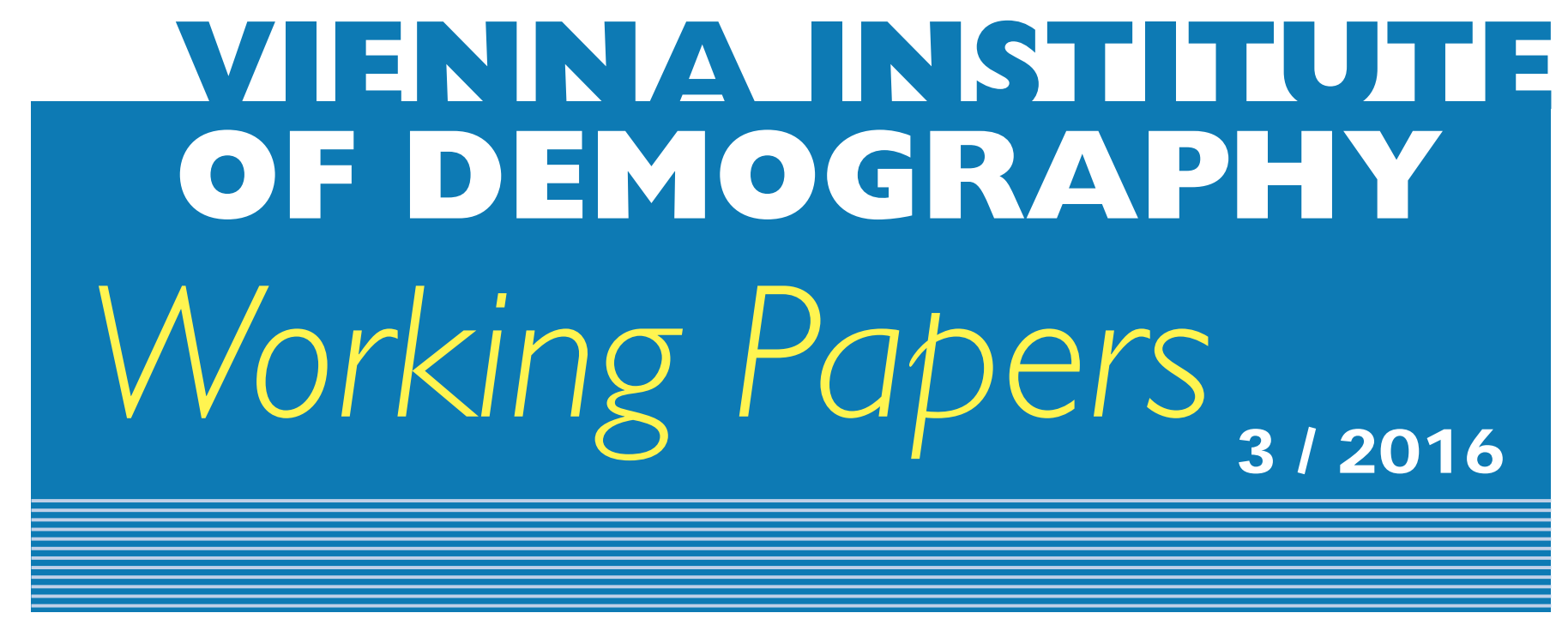

Ivan Frankovic, M ichael Kuhn and Stefan W rzaczek

\title{
Medical Care within an O LG Economy with Realistic Demography
}

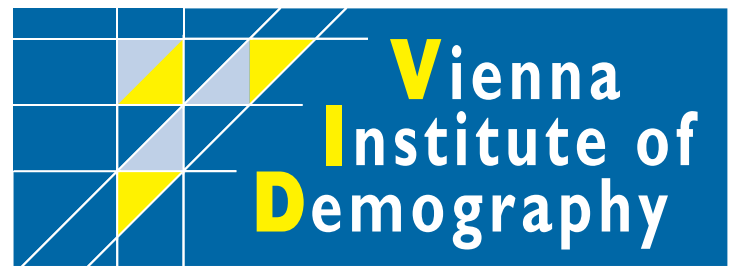

Vienna Institute of Demography Austrian Academy of Sciences

Welthandelsplatz 2 / Level 2

A-I020Vienna $\cdot$ Austria

E-Mail:vid@oeaw.ac.at

Website: www.oeaw.ac.at/vid

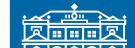

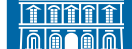

OAN Austrian Academy
of Sciences 


\begin{abstract}
We study the role of health care within a continuous time economy of overlapping generations subject to endogenous mortality. The economy consists of two sectors: final goods production and a health care sector, selling medical services to individuals. Individuals demand health care with a view to lowering mortality over their life-cycle. We derive the age-specific individual demand for health care based on the value of life as well as the resulting aggregate demand for health care across the population. We then characterize the general equilibrium allocation of this economy, providing both an analytical and a numerical representation. We study the allocational impact of a medical innovation both in the presence and absence of anticipation; and a temporary baby boom. We place particular emphasis on disentangling general equilibrium from partial equilibrium impacts and identifying the relevant transmission channels.
\end{abstract}

\title{
Keywords
}

Demographic change, life-cycle model, health care, health policy, medical change, overlapping generations, value of life

\section{Authors}

Ivan Frankovic, Wittgenstein Centre (IIASA, VID/ÖAW, WU), Vienna Institute of Demography, Austrian Academy of Sciences, Austria. Email: ivan.frankovic@oeaw.ac.at

Michael Kuhn, corresponding author, Wittgenstein Centre (IIASA, VID/ÖAW, WU), Vienna Institute of Demography, Austrian Academy of Sciences, Austria. Email: michael.kuhn@oeaw.ac.at

Stefan Wrzaczek, University of Vienna and Vienna University of Technology, Austria. Email: stefan.wrzaczek@tuwien.ac.at

\section{Acknowledgements}

Financial support for this research by the Austrian Science Fund under grant P 26184G11 is gratefully acknowledged. We are grateful to Friedrich Breyer, Ben Heijdra, Peter van der Meer and Peter Zweifel for helpful discussions and comments. The responsibility for any errors lies entirely with us. 


\title{
Medical Care within an OLG Economy with Realistic Demography
}

\author{
Ivan Frankovic, Michael Kuhn and Stefan Wrzaczek
}

\section{Introduction}

Although the impact of demographic and medical change on the sustainability of health care systems and the resulting need for reform have been the subject of empirical analyses for considerable time by now ${ }^{1}$ so far there have been only few theoretical attempts at the issues at stake. Undoubtedly this has to do with the complexity of such an analysis, which requires the integration of a life-cycle model with endogenous health and survival, a macro-economic overlapping generation (OLG) framework with a realistic population structure, and a two-sector model of production and health care.

A full account of the micro-macro feedback in terms of health policy is important both for an assessment of the impact of medical change and population change on micro-economic decision-making, and for tracing out the macro-economic dynamics. A long-standing literature has developed on the individual demand for health and health care over the life-course (e.g. Grossman 1972; Ehrlich and Chuma 1990; Ried 1998; Ehrlich 2000; Hall and Jones 2007; Kuhn et al. 2011, 2015; Dalgaard and Strulik 2014). This work is providing important insights as to how the demand for health and health care depends on the various prices; on the individual's wealth and income stream; on the cost of medical care; on the individual's socioeconomic background, such as education; on the interaction of health care choices with other life-cycle decision, such as the supply of labour; on market imperfections, e.g. within the annuity market; as well as on policies relating to health and other relevant domains. This literature typically takes a partial equilibrium stance by assuming an exogenous set of prices. Even if the response of the demand for health care to price changes is developed within these models, a failure to endogenise the response of prices to movements of supply and demand within the various markets may lead to erroneous conclusions, once it comes to an analysis of the impact of external changes on individual behaviour.

\footnotetext{
${ }^{1}$ See e.g. Breyer and Felder (2006) and Breyer et al. (2015) for Germany; Dormont et al. (2006) for France; Meara et al. (2004) and Shang and Goldman (2007) for the US; Karlsson and Klohn (2014) for Sweden; Zweifel et al. (2005) for a set of OECD countries; and European Union (2012) for the EU27. For an overview see Breyer et al. (2010).
} 
Many of the studies seeking to project the impact of demographic and technological trends on the share of health expenditure in GDP as well as on other macro-economic outcomes, notably economic growth, neglect the impact of the shifts in supply and demand on the price structure within the economy as well as the ensuing behavioural responses. Even if different "behavioural" scenarios are considered within such projections, a failure to endogenise the full feed-back process and how it emerges over time is likely to lead to biased forecasts. By now, a literature is emerging that integrates the impact of health care into general equilibrium analysis (e.g. Chakraborty 2004; Bhattacharya and Qiao 2007; Hall and Jones 2007; Yew and Chang 2014; Grossmann and Strulik 2014; Zhao 2014; Koijen et al. 2016; Kuhn and Prettner 2016; Schneider and Winkler 2016). However, much of this literature is based on rather stylised models of the life-cycle and the underlying mortality process, involving either a two period life-cycle or an assumption of perpetual youth (i.e. a constant mortality rate). Furthermore, many of the models either rely on a social planer approach or assume health care to evolve in a mechanical way, e.g. following a fixed fraction of income. Thus, the literature tends to abstract from the macro-economic effects induced by the changes in the age-structure that follow medical or policy change, and often refrains from a proper integration of the supply and the demand for health care. ${ }^{2}$

In this paper, we present an OLG model, involving an endogenous demand and supply of health care. The demand for health and health care is derived from utility maximisation within a life-cycle model with a realistic mortality pattern. Health care is provided within a medical sector, employing capital and labour, competing for resources with a final goods production sector. We characterise the optimal life-cycle allocation in terms of consumption and health care and show how it evolves with age, depending on the various prices, on the state of technology and population replacement (births). As one important determinant of the demand for health care, we characterise the value individuals attach to their survival, which will prove to be an important link between macro-economic changes and their impact on the micro-decisions. Solving the profit maximisation problem of perfectly competitive providers within the final goods and health care sectors, we can characterise the optimal structure of supply and factor demand as well as the aggregate dynamics. In particular, we derive an equation describing the dynamics of the aggregate demand for health care, which in analogy to

\footnotetext{
${ }^{2}$ Of the literature referenced above, Zhao (2014) is closest to our work in also considering a full general equilibrium model with a realistic life-cycle pattern and an endogenous choice of health care. The focus, however, lies on the impact of social security on health care spending, whereas our applications focus on the role of medical change and population change for the provision of health care.
} 
the aggregate Euler equation for consumption does not only depend on the weighted age-time trajectories at individual level but also on the turnover of the population.

We employ our model to analyse numerically the impact of medical progress and population change on the provision of health care. Based on a steady-state benchmark scenario that is calibrated to represent the US economy in the year 2003, we illustrate the importance of the micro-macro feedback by studying three numerical "experiments": the impact of a medical innovation which is either (i) unanticipated or (ii) anticipated; and (iii) the impact of a baby-boom. In the course of the analysis, we draw out both the macro-dynamics as well as the impact of the macro-economic feedback on the life-cycle allocation of consumption and health care, keeping track of different cohorts. The quasi-experimental character of our numerical analysis helps us to identify the salient transmission channels which need to be taken into account when trying to understand the impact of medical and demographic change on the provision of health care and on economic performance. This distinguishes our work from most of the other models (e.g. Zhao 2014, Koijen et al. 2016) which are calibrated to reflect the dynamics of the economy but render it much more difficult to disentangle the transmission channels from interfering time trends.

Our key findings include the following. Considering a medical innovation that improves the effectiveness of health care and raises life expectancy by a little more than 1 year, we find that health expenditure per capita increases by some $13 \%$, about 1 percentage point of which owing to an increase in the price for medical care, about 2 percentage points owing to the ageing of the population that is induced by the medical innovation, and the remaining 10 percentage points owing to an increase in individual demand. Although this is a substantive impact, we find that about half of the increase in individual demand that would be obtained otherwise is absorbed by the price increase in general equilibrium. With the health expenditure share in GDP increasing by some 1.7 percentage points, it may come as a surprise perhaps, that the level of GDP per capita itself remains unaffected. This is because the drop in the employment rate that comes with a disproportionate increase in survival amongst the retired population is neutralised by the accumulation of additional wealth that is induced by the increase in longevity and the prospect for individuals to purchase more effective health care in their old age. ${ }^{3}$ Indeed, if a medical innovation is fully anticipated, individuals actively defer the consumption of health care to post-innovation times, the ensuing increase in savings triggering a temporary economic boom. Finally, mortality reducing medical

\footnotetext{
${ }^{3}$ This is consistent with empirical evidence provided by De Nardi et al. (2010).
} 
innovations tend to come with a reduction in the value of life over large parts of the life-course. On the one hand, this reflects a reduction in consumption levels; on the other hand, it implies that the price of medical care per life-year gained has fallen, a result that is in line with empirical evidence (Cutler et al. 1998).

A temporary baby-boom triggers two partially offsetting ripples in the per capita consumption of health care: The shift in age-structure from an initially young to an ageing population is reflected in a movement of per capita health care that first involves a slump and then a peak. Super-imposed on these age-structure effects, there are changes in the level of individual demand for care: low prices of health care during the initial labour boom induced by the large incoming cohorts trigger an increase in the individual consumption of health care. This is followed by a slump as the entry of the baby boomers into retirement boosts social security and Medicare taxes, and as the demand for health care by the ageing baby boom cohorts drives up the price for care. These movements are also reflected in the experience of different cohorts: while the elderly who are still alive at the time of the baby boom tend to benefit in terms of greater consumption and health care, the baby boom cohorts themselves face a reduction of consumption and health care once they enter old age.

The remainder of the paper is structured as follows: The following section is devoted to a presentation of the model; sections 3 and 4 solve for and characterise the individual life-cycle allocation and the general equilibrium of the economy, respectively; section 5 presents the numerical analysis before section 6 wraps up. Some of the proofs have been relegated to an Appendix.

\section{The Model}

We consider an OLG model in which individuals choose consumption and health care over their life-course. Individuals are indexed by their age $a$ at time $t$, with $t_{0}=t-a$ denoting the birth year of an individual aged $a$ at time $t$. At each age, individuals are subject to a mortality risk, where $S(a, t)=\exp \left[-\int_{0}^{a} \mu(\widehat{a}, h(\widehat{a}, \widehat{t}), M(\widehat{t})) d \widehat{a}\right]$ is the survival function at $(a, t)$, with $\mu(a, h(a, t), M(t))$ denoting the force of mortality. Following Kuhn et al. (2010, 2011, 2015) we assume that mortality can be lowered by the consumption of a quantity $h(a, t)$ of health care. In addition, we assume that mortality depends on the state of the medical technology $M(t)$ at time $t$. More specifically, we 
assume that the mortality rate $\mu(a, h(a, t), M(t))$ satisfies

$$
\begin{aligned}
\mu(a, h(a, t), M(t)) & \in(0, \tilde{\mu}(a, t)] \quad \forall(a, t) ; \\
\mu_{h}(\cdot) & <0, \mu_{h h}(\cdot)>0 ; \\
\mu_{h}(a, 0, M(t)) & =-\infty, \mu_{h}(a, \infty, M(t))=0 \quad \forall(a, t) ;
\end{aligned}
$$

where $\tilde{\mu}(a, t)=\mu(a, 0, M(t))$ is the "natural "mortality rate for an individual aged $a$ at time $t$ when no health care is consumed. By purchasing health care, an individual can lower the instantaneous mortality rate, and can thereby improve survival prospects, but can only do so with diminishing returns. ${ }^{4}$

In regard to medical technology, we assume the following properties

$$
\mu_{M}(\cdot) \leq 0, \mu_{M M}(\cdot) \geq 0, \mu_{h M}(\cdot) \gtreqless 0 \quad \forall(a, t),
$$

thus admitting for two possible cases: (i) a situation in which medical technology contributes toward reductions in mortality $\left(\mu_{M}(\cdot)<0\right)$ with (weakly) decreasing returns. We leave it open, however, whether for any given positive level of health care, $h(a, t)>0$, medical technology is complementing the consumption of health care $\left(\mu_{h M}(a, h(a, t), M(t)) \leq 0\right)$ or substituting it $\left(\mu_{h M}(a, h(a, t), M(t))>0\right)$. (ii) As a special case, we allow that medical technology has no direct impact on mortality but merely changes the production of health care $\left(\mu_{M}(\cdot)=0\right) .^{5}$

Individuals enjoy period utility $u(c(a, t))$ from consumption $c(a, t)$. Period utility is increasing and concave: $u_{c}(\cdot)>0, u_{c c}(\cdot) \leq 0$. In addition, we assume the Inada condition $u_{c}(0)=+\infty$. Individuals maximise their expected life-cycle utility

$$
\max _{c(a, t), h(a, t)} \int_{0}^{\omega} e^{-\rho a} u(c(a, t)) S(a, t) d a
$$

by choosing a stream of consumption and health care on the interval $[0, \omega]$, with $\omega$ denoting the maximal possible age and $\rho \geq 0$ denoting the rate of time preference. ${ }^{6}$ The individual faces as constraints the dynamics of survival and the dynamics of individual

\footnotetext{
${ }^{4}$ Zweifel et al. (2005) provide empirical evidence of decreasing returns to health expenditure in the reduction of mortality. The decreasing returns assumption is also reflected in other empirical work on the relationship between health care and mortality (e.g., Cremieux et al. 1999, Lichtenberg 2004, Hall and Jones 2007, Baltagi et al. 2012).

${ }^{5}$ In principle, some cost saving medical technologies may be conceived of that lead to greater mortality $\left(\mu_{M}(\cdot)>0\right)$. For the purpose of this analysis we abstract from this third type.

${ }^{6}$ Note that from the individual's perspective age $a \in[0, \omega]$ and time $t=t_{0}+a \in\left[t_{0}, t_{0}+\omega\right]$ are identical. Thus, we could also write life-cycle utility as $\int_{t_{0}}^{t_{0}+\omega} e^{-\rho t} u(c(a, t)) S(a, t) d t$.
} 
assets $k(a, t)$, as described by the system

$$
\begin{aligned}
\dot{S}(a, t)= & -\mu(a, h(a, t), M(t)) S(a, t), \\
\dot{k}(a, t)= & r(t) k(a, t)+l(a) w(t)-c(a, t) \\
& -\phi(a, t) p_{H}(t) h(a, t)-\tau(a, t)+\pi(a, t)+d_{H}(t)+s(t),
\end{aligned}
$$

with the boundary conditions

$$
\begin{aligned}
& S\left(0, t_{0}\right)=1, \quad S\left(\omega, t_{0}+\omega\right)=0 \\
& k\left(0, t_{0}\right)=k\left(\omega, t_{0}+\omega\right)=0 .
\end{aligned}
$$

Here, (2) describes the reduction of survival according to the force of mortality. According to (3) an individual's stock of assets $k(a, t)$ (i) increases with the return on the current stock, where $r(t)$ denotes the interest rate at time $t$; (ii) increases with earnings $l(a) w(t)$, where $w(t)$ denotes the wage rate at time $t$, and where $l(a)$ denotes an individual's effective age-dependent labour supply; (iii) decreases with consumption, the price of consumption goods being normalised to one; (iv) decreases with private health expenditure, $\phi(a, t) p_{H}(t) h(a, t)$, where $p_{H}(t)$ denotes the price for health care, and where $\phi(a, t)$ denotes an $(a, t)$-specific rate of coinsurance; $(\mathrm{v})$ decreases with an $(a, t)$ specific tax, $\tau(a, t)$; (vi) increases with $(a, t)$-specific benefits $\pi(a, t)$; (vii) increases with possible dividends paid by health-care providers, $d_{H}(t)$; and (viii) increases with a transfer $s(t)$ by which the government redistributes accidental bequests in a lump-sum fashion. Here, we follow Ludwig et al. (2012) and Zhao (2014) by considering a setting without an annuity market. ${ }^{7}, 8$ We assume that the survival function is bounded between 1 at birth and 0 at the maximum feasible age $\omega$ [see (4)], and that individuals enter and leave the life-cycle without assets [see (5)].

Denoting by $B(t-a)$ the size of the birth cohort at $t_{0}=t-a$ and aggregating over the age-groups who are alive at time $t$ we obtain the following expressions for the population size, aggregate capital stock, aggregate effective labour supply, aggregate consumption, aggregate demand for health care, aggregate fiscal income from taxation,

\footnotetext{
${ }^{7}$ This is well in line with evidence that few individuals annuitise their wealth (e.g. Warwshawsky 1988, Reichling and Smetters 2015). Hansen and Imrohoroglu (2008) show that the empirically relevant hump-shaped life-cycle profiles of consumption can be consistently explained within a life-cycle model only when assuming that annuity markets are assumed to be absent (or severely imperfect).

${ }^{8}$ We have also considered a specification with imperfect annuities yielding a return $r(t)+\theta \bar{\mu}(a, t)$, where $\theta \in[0,1]$ and where $\bar{\mu}(a, t)=\mu\left(a, h^{*}(a, t), M(t)\right)$ is the expected mortality, given the equilibrium level of health care $h^{*}(a, t)$. Following Heijdra and Mierau (2012) in considering a scenario with $\theta=0.7$, we obtain qualitatively similar results to those reported in this paper.
} 
and aggregate transfer payments, each at time $t$ :

$$
\begin{aligned}
N(t) & =\int_{0}^{\omega} S(a, t) B(t-a) d a, \\
K(t) & =\int_{0}^{\omega} k(a, t) S(a, t) B(t-a) d a, \\
L(t) & =\int_{0}^{\omega} l(a, t) S(a, t) B(t-a) d a, \\
C(t) & =\int_{0}^{\omega} c(a, t) S(a, t) B(t-a) d a, \\
H(t) & =\int_{0}^{\omega} h(a, t) S(a, t) B(t-a) d a, \\
\Upsilon(t) & =\int_{0}^{\omega} \tau(a, t) S(a, t) B(t-a) d a, \\
\Pi(t) & =\int_{0}^{\omega} \pi(a, t) S(a, t) B(t-a) d a .
\end{aligned}
$$

The economy consists of a manufacturing sector and a health care sector. In the competitive manufacturing sector a final good is produced by employment of capital $K_{Y}(t)$ and labour $L_{Y}(t)$ according to a neoclassical production function $Y\left(K_{Y}(t), A(t) L_{Y}(t)\right)$, with $A(t)$ measuring the state of labour augmenting technology. A manufacturer's profit can then be written as

$$
V_{Y}(t)=Y\left(K_{Y}(t), A(t) L_{Y}(t)\right)-w(t) L_{Y}(t)-[\delta+r(t)] K_{Y}(t),
$$

where $\delta$ denotes the depreciation rate of capital. Note that the presence of perfect competition together with a neoclassical production function implies $V_{Y}(t)=0$ in equilibrium.

Health care goods and/or services are produced by employment of labour $L_{H}(t)$, capital $K_{H}(t)$, and technology $M(t)$ according to the production function $F\left(M(t), K_{H}(t), L_{H}(t)\right) .{ }^{9}$ Recalling the price for health care $p_{H}(t)$, the profit of a health care provider is then given by

$$
\begin{aligned}
V_{H}(t)= & p_{H}(t) F\left(M(t), K_{H}(t), L_{H}(t)\right)-w(t) L_{H}(t) \\
& -[\delta+r(t)] K_{H}(t),
\end{aligned}
$$

where we assume that capital depreciates at the same rate across both sectors. Allowing

\footnotetext{
${ }^{9}$ This generalises, by the capital and technology component, the formulation in Schneider and Winkler (2010) and Kuhn and Prettner (2016).
} 
that the production function of health care $F(\cdot)$ may not be neoclassical, we let $V_{H}(t)=$ $d_{H}(t) N(t)$ denote the redistribution of (potential) profits through dividends.

The government and/or a third-party payer (e.g. a health insurer) raise taxes (or contribution rates, e.g. insurance premiums) for the purpose of co-financing health care at the rate $1-\phi(a, t)$ and of paying out transfer payments $\pi(a, t)$. More specifically, $\pi(a, t)$ may refer to pension benefits, implying that

$$
\pi(a, t)=\left\{\begin{array}{l}
0 \Leftrightarrow a<a_{R} \\
\pi \geq 0 \Leftrightarrow a \geq a_{R}
\end{array}\right.
$$

with $\pi$ a uniform pension benefit and $R$ the retirement age. In such a setting we would also have

$$
l(a, t)=\left\{\begin{array}{l}
l(a, t) \geq 0 \Leftrightarrow a<a_{R} \\
0 \Leftrightarrow a \geq a_{R}
\end{array}\right.
$$

Likewise, $\tau(a, t)$ are age-specific taxes. We could distinguish these into taxes used to finance health care payments (or health insurance premiums), $\tau_{H}(a, t)$, and social security contributions, $\tau_{\Pi}(a, t)$, where $\tau(a, t)=\tau_{H}(a, t)+\tau_{\Pi}(a, t)$. Furthermore, we could, in principle distinguish between lump-sum and labour income taxes, $\tau_{j}(a, t)=$ $\widehat{\tau}_{j}(a, t) l(a, t) w(t)$, with $j=H, \Pi$. As long as we assume a unified government budget and an exogenous labour supply, it is sufficient to consider $\tau(a, t)$.

Assuming that the government budget must be balanced within each period $t$ we obtain the constraint

$$
\int_{0}^{\omega}\left\{\begin{array}{c}
{[1-\phi(a, t)] p_{H}(t) h(a, t)} \\
+\pi(a, t)-\tau(a, t)
\end{array}\right\} S(a, t) B(t-a) d a=0 .
$$

We can then write

$$
\Upsilon(t)-\Pi(t)=p_{H}(t) \int_{0}^{\omega}[1-\phi(a, t)] h(a, t) S(a, t) B(t-a) d a
$$

as the publicly financed amount of health expenditure. Finally, we assume that

$$
s(t)=\frac{\Upsilon_{B}(t)}{N(t)}
$$

where

$$
\Upsilon_{B}(t)=\int_{0}^{\omega} \mu(a, t) k(a, t) N(a, t) d a
$$


are total accidental bequests.

\section{Individual Life-Cycle Optimum}

In Appendix A1 we show that the solution to the individual life-cycle problem is described by the following two sets of conditions

$$
\begin{aligned}
\frac{u_{c}(c(a, t))}{e^{-\rho(\widehat{a}-a)} u_{c}(c(\widehat{a}, t+\widehat{a}-a))} & =e^{\int_{a}^{\widehat{a}} r(t+\widehat{a}-a) d \widehat{a}} \frac{S(\widehat{a}, t+\widehat{a}-a)}{S(a, t)}, \\
-\mu_{h}(a, t) \psi(a, t) & =\phi(a, t) p_{H}(t) \quad \forall(a, t),
\end{aligned}
$$

describing the optimal pattern of consumption $c(a, t)$ and the demand for health care $h(a, t)$, respectively, of an individual aged $a$ at time $t$. Condition (15) is the well-known Euler equation, requiring that the marginal rate of intertemporal substitution between consumption at any two ages/years $(a, t)$ and $(\widehat{a}, t+\widehat{a}-a)$ equals the compound interest, which, in the absence of an annuity market, is weighted with the conditional probability of surviving to $(\widehat{a}, t+\widehat{a}-a)$.

Condition (16) requires that at each $(a, t)$ the marginal value of health care, $-\mu_{h}(a, t) \psi(a, t)$, equals its effective price, $\phi(a, t) p_{H}(t)$. The marginal value of health care is given by the marginal effect of health care on mortality, $-\mu_{h}(a, t)$, weighted with the private value of life (VOL). The private VOL is defined by

$$
\psi(a, t):=\int_{a}^{\omega} \frac{u(c(\widehat{a}, t+\widehat{a}-a))}{u_{c}(\cdot)} R(\widehat{a}, a) d \widehat{a},
$$

with

$$
R(\widehat{a}, a):=\exp \left[-\int_{a}^{\widehat{a}} r(t+\widehat{\widehat{a}}-a) d \widehat{\widehat{a}}\right],
$$

and amounts to the discounted stream of consumer surplus, $u(\cdot) / u_{c}(\cdot)$ taken over the expected remaining life-course $[a, \omega] .^{10}$

The dynamics of consumption are described by (for a derivation see Appendix A1)

$$
\dot{c}=\frac{u_{c}}{u_{c c}}(\rho-r+\mu) .
$$

Noting that $u_{c c}<0$, it is readily seen that consumption tends to increase over the

\footnotetext{
${ }^{10}$ The VOL as we calculate it here differs from the typical representation of the value of a statistical life as e.g. in Shepard and Zeckhauser (1984), Rosen (1988), Johansson (2002), or Murphy and Topel (2006) in as far as (i) the discount factor does not include the mortality rate; and (ii) the VOL does not include the current change to the individual's wealth, $l w-c-h-\tau+\pi+d_{H}+s$. Both of these features are due to the absence of an annuity market.
} 
life-cycle if and only if $r-\rho>\mu$. In the absence of an annuity market, the uninsured mortality risk imposes a downward drag on consumption over the life-cycle and implies that consumption will eventually decrease with age when mortality $\mu$ grows sufficiently high.

The dynamics of health care are described by (for a derivation see Appendix A1)

$$
\dot{h}=\frac{-1}{\mu_{h h}}\left[\mu_{h a}+\mu_{h M} \dot{M}+\mu_{h}\left(\frac{\dot{\psi}}{\psi}-\frac{\dot{p}_{H}}{p_{H}}-\frac{\dot{\phi}}{\phi}\right)\right] .
$$

Noting that $\mu_{h h}>0$, the demand for health care evolves with age/time under the influence of three forces: (i) the changing effectiveness of health care with age $\mu_{h a}$, a stronger (weaker) effectiveness with age, $\mu_{h a}<0(>0)$ implying an increase (decrease) in health care; (ii) the change in the effectiveness of health care owing to medical change; (iii) the rate of change in the VOL, a decrease implying a reduction in health care; (iv) the rate of change in medical prices, an increase implying a reduction in health care; and (v) the rate of change of the coinsurance rate.

Differentiating (17) with respect to age-time, we obtain the dynamics of the private VOL as

$$
\dot{\psi}(a, t)=r(t) \psi(a, t)-\frac{u(c(a, t))}{u_{c}(c(a, t))} .
$$

Thus, the private VOL increases with the interest rate and declines over time as the consumer surplus from a life-year lived is written off.

\section{General Equilibrium}

Perfectly competitive firms in the production sector choose labour $L_{Y}(t)$ and capital $K_{Y}(t)$ so as to maximise period profit (10). The first-order conditions imply

$$
\begin{aligned}
r(t) & =Y_{K_{Y}}(t)-\delta \\
w(t) & =Y_{L_{Y}}(t),
\end{aligned}
$$

i.e. the factor prices are equalised with their respective marginal products.

Likewise, perfectly competitive providers of health care choose labour $L_{H}(t)$ and capital $K_{H}(t)$ so as to maximise period profit (11). From the first-order condition we 
obtain

$$
\begin{aligned}
r(t) & =p_{H}(t) F_{K_{H}}(t)-\delta \\
w(t) & =p_{H}(t) F_{L_{H}}(t) .
\end{aligned}
$$

Combining these conditions with (22) and (23) we obtain

$$
p_{H}(t)=\frac{Y_{L_{Y}}(t)}{F_{L_{H}}(t)}=\frac{Y_{K_{Y}}(t)}{F_{K_{H}}(t)}
$$

implying that capital and labour inputs are distributed across the production and health care sector in a way that equalises the marginal rate of transformation (i.e. the relative output gain in production as compared to the output loss in health care from re-allocating one factor unit from health care into production) with the price for health care. The higher the latter, the greater the marginal rate of transformation, implying that more workers will be allocated to the health care sector. With appropriate Inada conditions, $Y_{L_{Y}}\left(K_{Y}, 0\right)=Y_{K}=\left(0, A L_{Y}\right)=\infty$ and $F_{L_{H}}(M, K, 0)=F_{K}\left(M, 0, L_{H}\right)=\infty$ we always have an interior allocation with $L_{H}(t)=L(t)-L_{Y}(t) \in(0, L(t))$ and $K_{H}(t)=K(t)-K_{Y}(t) \in(0, K(t))$.

\subsection{Market Clearance and General Equilibrium}

Our setting involves four markets: two input markets for capital and labour, respectively; and two output markets for health care and for final goods, respectively. From the four market clearing conditions

$$
\begin{aligned}
K_{Y}(t)+K_{H}(t) & =K(t), \\
L_{Y}(t)+L_{H}(t) & =L(t) \\
F(t) & =H(t), \\
Y(t) & =C(t)+\dot{K}(t)+\delta K(t),
\end{aligned}
$$

we obtain a set of equilibrium prices $\left\{r^{*}(t), w^{*}(t), p_{H}^{*}(t)\right\}$ as well as the level of net capital accumulation $\dot{K}(t)$. We provide a more detailed description of the general equilibrium structure in Appendix A2. 


\subsection{Dynamics}

The dynamics of the economy are described by the system (for a derivation see Appendix A3)

$$
\begin{aligned}
\dot{N}(t) & =B(t)-\int_{0}^{\omega} \mu(a, t) N(a, t) d a, \\
\dot{K}(t) & =Y(t)-C(t)-\delta K(t), \\
\dot{C}(t) & =\int_{0}^{\omega} \frac{u_{c}}{u_{c c}}[\rho-r(t)+\mu(a, t)] N(a, t) d a+\Omega_{C}(t), \\
\dot{H}(t) & =\int_{0}^{\omega} \dot{h}(a, t) N(a, t) d a+\Omega_{H}(t),
\end{aligned}
$$

depicting the evolution of the population, the aggregate capital stock, aggregate consumption and the aggregate demand for health care, respectively. For an exogenous number of births, $B(t)$, health care is shaping population growth by modifying mortality and, thus, the aggregate number of deaths at each point in time. Capital is accumulated according to the difference between final goods output, on the one hand, and consumption and capital replacement, on the other.

Aggregate consumption evolves according to the aggregate Euler equation, which is composed of the individual Euler equation (19), aggregated over the various cohorts, and of the generational turnover

$$
\begin{aligned}
\Omega_{C}(t) & :=B(t) c(0, t)-C^{\dagger}(t), \\
\text { with } \quad C^{\dagger}(t) & :=\int_{0}^{\omega} \mu(a, t) c(a, t) N(a, t) d a,
\end{aligned}
$$

expressing the difference between the aggregate consumption of the newborn cohort and the aggregate consumption of the deceased, $C^{\dagger}(t)$ (see e.g. Heijdra and Mierau 2012, Mierau and Turnovsky 2014, Kuhn and Prettner 2015).

The evolution of the aggregate demand for health care, as described by (30), follows the same pattern as aggregate consumption. Specifically, it is composed of (i) the $(a, t)$-change in an individual's demand for health care, aggregated over the various age-groups, and the generational turnover in regard to health care

$$
\begin{aligned}
\Omega_{H}(t) & :=B(t) h(0, t)-H^{\dagger}(t), \\
\text { with } \quad H^{\dagger}(t) & :=\int_{0}^{\omega} \mu(a, t) h(a, t) N(a, t) d a,
\end{aligned}
$$


corresponding to the difference between the aggregate demand for health care by the newborn cohort and the aggregate demand for health care by the deceased. The generational turnover $\Omega_{H}(t)$ term provides a neat summary of the impact of demographic change on the development of the aggregate demand for health care. Notably, the term $H^{\dagger}(t)$ looks beyond death. While the process of dying increases the aggregate usage of health care, this resource use is "written of" after the individual has died. The increasing resource use with approaching death is measured in ex-ante terms by the change in the individual demand for health care, $h(a, t)$. To the extent that health care is particularly effective in the presence of high mortality risk, its usage is bound to increase with mortality and, thus, with the statistical proximity to death (see e.g. Zweifel et al. 1999). This said, the demand level $h(a, t)$ in our model has been derived as an ex-ante optimum for a representative consumer. For this reason, $h(a, t)$ is measuring the average resource use across survivors and decedents within age group $a$ at time $t .{ }^{11}$

Combining (27) and (30) and rearranging, we obtain a convenient expression for the growth rate of per capita health expenditure

$$
\begin{aligned}
g_{H / N}(t): & =\frac{H(t)}{H(t)}-\frac{N(t)}{N(t)} \\
= & \int_{0}^{\omega} \frac{\dot{h}(a, t)}{h(a, t)} \frac{h(a, t) N(a, t)}{H(t)} d a \\
& +\frac{B(t)}{N(t)}\left[\frac{h(0, t)}{H(t) / N(t)}-1\right] \\
& -\frac{D(t)}{N(t)} \int_{0}^{\omega}\left[\frac{h(a, t)}{H(t) / N(t)}-1\right] \frac{\mu(a, t) N(a, t)}{D(t)} d a,
\end{aligned}
$$

with $D(t):=\int_{0}^{\omega} \mu(a, t) N(a, t) d a$ denoting the number of deaths in period $t$. The growth rate of per capita health expenditure is, thus, composed of three parts: (i) the growth rate of individual health expenditure averaged across all age-groups, with the age specific expenditure shares used as weights; (ii) the product of the birth rate, $B(t) / N(t)$, and a measure of the excess expenditure of the newborn; and (iii), entering negatively, the product of the death rate, $D(t) / N(t)$, and a measure of the average

\footnotetext{
${ }^{11}$ While our model can therefore be calibrated to the average resource use at macro-level, its power to explain the individual cost of health care is limited by the representative consumer approach, for which it is impossible to disentangle whether average health care costs within an age-group are explained by a high share of this cohort being close to death or by an age-effect proper. Such an exercise, performed in empirical studies (e.g. Zweifel et al. 1999) would require a model with individual heterogeneity in regard to both health state and age.
} 
excess spending by the dying, with the age-shares in the total death toll used as weights. Provided that health expenditure levels among the young fall short of the per capita value, a higher birth rate is, thus, prone to depress the growth rate of per capita expenditure. Likewise, given that average health expenditure of the dying exceeds the per capita value, a high death rate is also exerting a downward pressure on per capita expenditure growth. Here, the positive correlation of mortality $\mu(a, t)$ and expenditure $h(a, t)$ suggests that the integral term in (iii) is, indeed, positive.

Typically, the next step would involve the analysis of a balanced growth path, for which the population grows at some constant rate $N(t) / N(t)=n$ and the aggregate variables grow at some rate $\dot{K}(t) / K(t)=\dot{C}(t) / C(t)=\dot{H}(t) / H(t)=n+g$, with $g$ denoting the rate of per capita growth, typically equivalent to the rate of technical progress. In this study we refrain from such an exercise because under endogenous mortality it is not clear a priori whether a balanced growth path is prone to exist. The reason is that, as long as the demand for health care increases over time, the ongoing reduction in mortality keeps on changing the aggregate Euler equations, both for consumption and for health care. These shifts are consistent with constant growth rates if and only if they take on a very particular, and typically unrealistic, form.

More generally, one could argue that balanced growth paths themselves constitute an abstraction designed to analyse the very long run prospects of an economy. For our analysis being particularly focused on the dynamic effects of medical change and population change, such a long-run perspective is not exceedingly interesting. Finally, with our numerical framework allowing us to characterise the dynamics of the economy in some detail, we will focus in the following on a numerical analysis of the dynamics without assuming balanced growth.

\section{Numerical Analysis}

Following a description of our numerical analysis, we present the outcomes for four scenarios, consisting of a benchmark and three numerical experiments. The benchmark features a realistic economy calibrated to US data, reflecting the year 2003. The experiments involve (i) the impact of an unanticipated medical advance, leading to a reduction in mortality; (ii) the impact of the same advance when it is anticipated; and (iii) the impact of an (unanticipated) baby boom. 


\subsection{Specification of the Numerical Analysis}

The main components of our numerical model are specified as follows.

\section{Demography}

With model time progressing in single years, individuals enter the model economy at age 20 and can live up to a maximum age 100. In our model, a birth at age 20 implies that $\omega=80$. Population growth is partly endogenous due to endogenous mortality but also exogenous due to a fixed growth rate of births $\nu=1.3 \%$, which is calibrated to match the elderly share of the adult (20 years and older) US population, equalling to $17.6 \%$ according to the US Census 2000. Due to the exogenous path of births, our results will not be confounded by a variation in birth numbers across the experiments.

\section{Mortality}

The force of mortality $\mu$ is endogenously determined in the model, depending on health care, $h$, as a decision variable; an exogenous level of medical technology, $M$; and an exogenous age-dependent base mortality, $\widetilde{\mu}(a)$. As not all reductions in mortality can be attributed to health expenses or technological progress (see e.g. Hall and Jones 2007), we introduce an exogenous factor $I(a)$ that captures changes in age-dependent mortality rates due to exogenous circumstances. Following Kuhn et al. (2011, 2015) we formulate

$$
\mu(a, t)=\widetilde{\mu}(a) \cdot\left(I(a)-\eta(a)[h(a, t) \cdot M(t)]^{\epsilon(a)}\right),
$$

where $\eta(a)$ and $\epsilon(a)$ are parametric functions that reflect decreasing efficiency of health care with age. The base mortality reflects a mortality profile that is higher in level (to a sufficient extent) than the US mortality in the year 2003, which we aim to replicate in the calibration. For this purpose we chose mortality rates for the year 1950 in the US, as reported in the Human Mortality Database (HMD) providing single year death rates, see Figure 1. The age-dependent parametric functions $\eta(a), \epsilon(a)$ and $I(a)$ are chosen to approximate the US 2003 per-capita health expenditures, reported in the National Health Expenditure Accounts (NHEA), as provided by the Centers for Medicare \& Medicaid services (CMS) and the age-specific elasticities of mortality with respect to health expenditures estimated by Hall and Jones (2007). We normalise the state of medical technology to the year 2003 and, thus, set $M(t) \equiv 1$ in the benchmark case. 


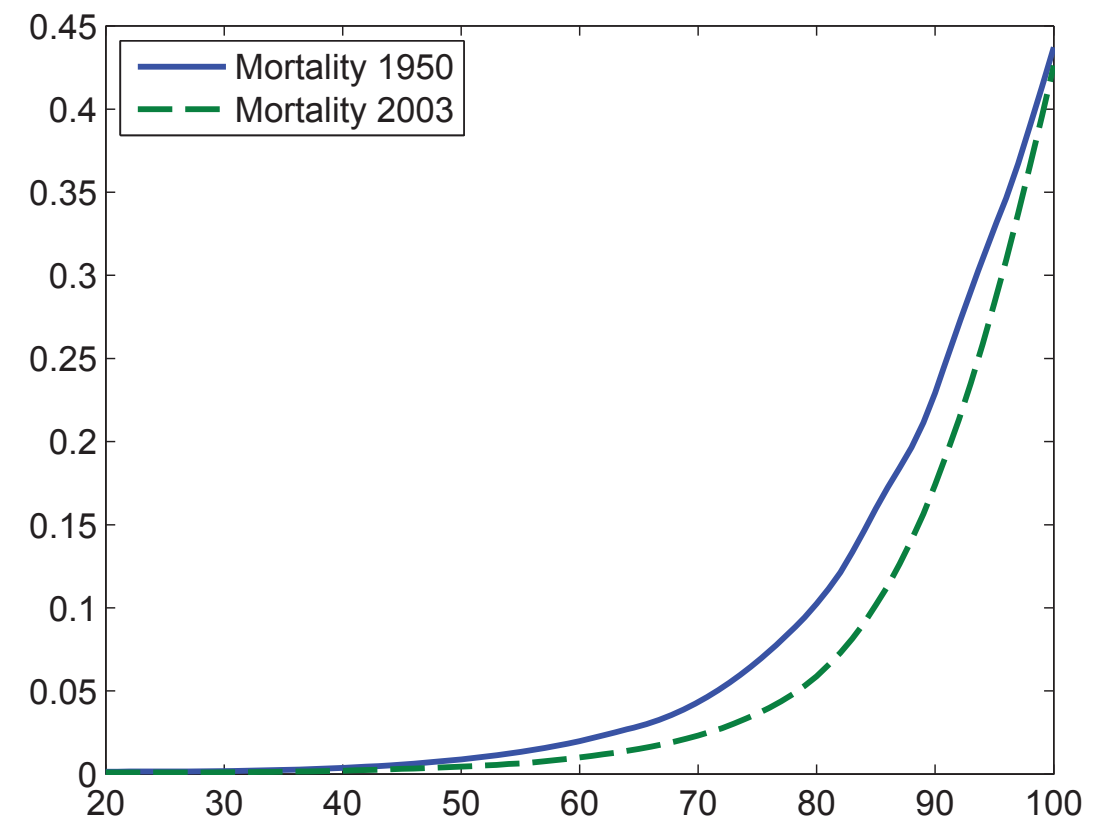

Figure 1: Force of mortality for 1950 and 2003 in the US (HMD)

\section{Utility}

Following the bulk of the literature, we assume instantaneous utility to be given by

$$
u(a, t)=b+\frac{\left(c(a, t)-c_{0}\right)^{1-\sigma}}{1-\sigma}
$$

with $b=8$ and $\sigma=1.75$. Here, $c_{0}=\$ 11000$ is an exogenous minimal consumption level. ${ }^{12}$ In addition, we assume a rate of time preference $\rho=2 \%$.

\section{Effective labour supply and income}

We proxy the effective supply of labour by an age-specific income schedule (see Figure 2), constructed from 2003 earnings data, as contained in the Current Population Survey (CPS) provided by the Bureau of Labor Statistics (BLS). We rescale the schedule such that the employment-population ratio $L(t) / N(t)$ matches the empirical value of $62 \%$ for the US in 2003 as reported by the BLS. Individuals at the age 65 or older are assumed to have no income from labour but receive a fixed social security pension for the remainder of their lifetime, as detailed further on below.

\footnotetext{
${ }^{12}$ Dollar values are, throughout the whole paper, to be interpreted as year 2003 Dollars.
} 


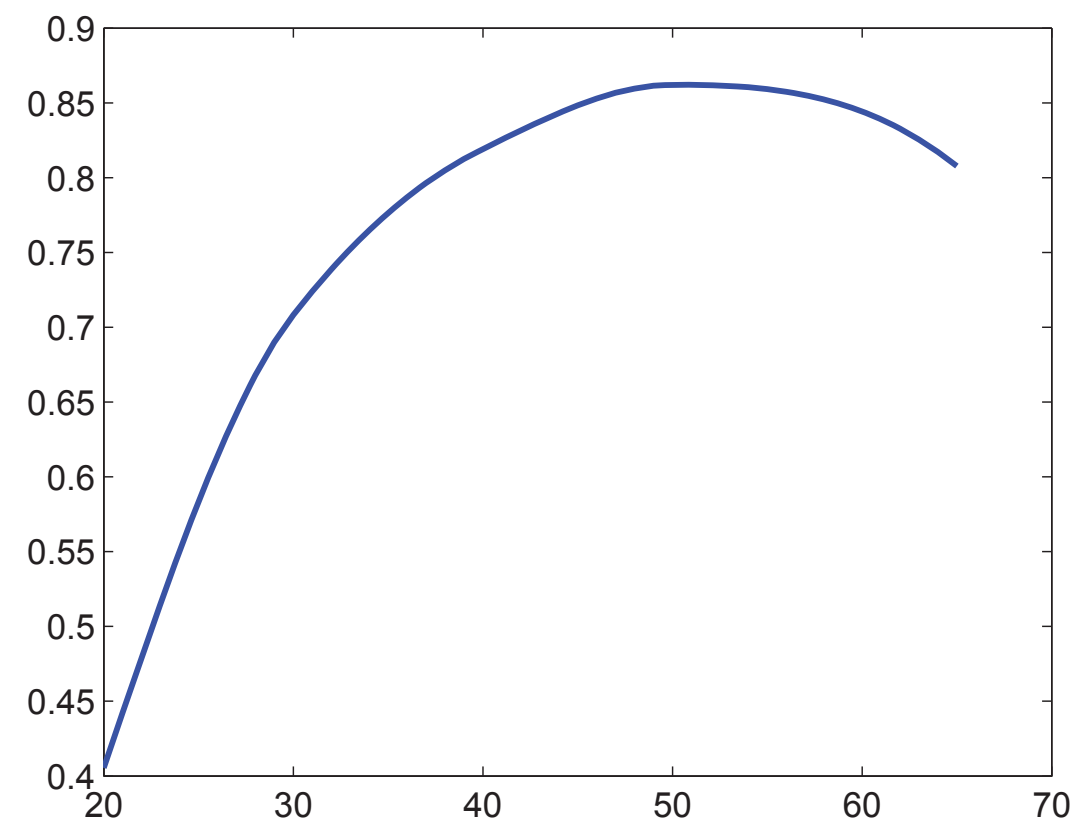

Figure 2: Age-specific labour employment schedule

\section{Production}

There are two production functions in the model. Production of the final good is described by

$$
Y(t)=K_{Y}(t)^{\alpha}\left(A(t) L_{Y}(t)\right)^{1-\alpha},
$$

where $K_{Y}(t)$ and $L_{Y}(t)$ denote capital and labour in final good production, where $L_{Y}(t)$ is the workforce working in this sector, and where $A(t)$ is an exogenous technology index. $A(t)$ is calibrated so that $l(50) w(t)$ matches the average earnings of a 50 -year old in 2003; the elasticity of capital $\alpha$ is chosen to be $1 / 3$.

The health care sector produces medical goods and services that individuals purchase with a view to lowering their mortality. Its production is given by

$$
F(t)=M(t)^{\gamma} K_{H}(t)^{\beta}\left(L_{H}(t)\right)^{1-\beta},
$$

where $K_{H}(t)$ and $L_{H}(t)$ denote capital and labour in this sector. The elasticity of capital $\beta$ is set to $\frac{1}{5}$, following Donahoe (2000). ${ }^{13}$ Assuming the elasticity of labour to be $1-\beta=\frac{4}{5}$ implies that there are no profits in the health care sector, such that

\footnotetext{
${ }^{13}$ Donahoe (2000) states that capital intensity per worker is approximately twice as large as in the non-health sector compared to the health sector. Using (48) and (49) in Appendix A5 we obtain $\beta=\frac{1}{5}$ for $\alpha=\frac{1}{3}$.
} 
$V_{H}(t)=d_{H}(t) \equiv 0$. For the purpose of this analysis, we assume the absence of a productivity effect of technology $M(t)$, i.e. $\gamma=0$. Finally, we assume a rate of capital depreciation equal to $\delta=0.05$.

\section{Health insurance, Medicare and Social Security}

Health expenditures are subsidised through two different sources: (a) private health insurance with coinsurance rate $\phi_{P}$ and (b) Medicare for the elderly (available after retirement) with coinsurance rate $\phi_{M C}$. Private health insurance is financed through a "risk-adequate" premium equal to the expected health expenditure covered by the insurance for an individual at a given time and age. It is thus given by $\tau_{P}=\left[1-\phi_{P}(a, t)\right] p_{H}(t) h^{*}(a, t)$, where $h^{*}(a, t)$ denotes the equilibrium demand for health care at $(a, t)$. Following Zhao (2014) we assume that $70 \%$ of the US workforce is health insured, with $70 \%$ of expenses being covered. Thus, we assume that $51 \%$ of health expenditures are paid out-of-pocket on average among the working population. Zhao (2014) states that $35 \%$ of the elderly have health insurance with a coverage of $30 \%$, leading to average health insurance subsidies of $10.5 \%$. Medicare is financed through a payroll tax, with the rate $\hat{\tau}_{M C}$ being endogenously determined such that the Medicare budget constraint holds. We assume that Medicare covers $38 \%$ of the health expenses of the elderly ${ }^{14}$. This results in $51.5 \%$ out-of-pocket expenditures for the elderly. In total, the out-of-pocket share of health expenses paid by the individual is

$$
\phi=\left\{\begin{array}{c}
0.51 \text { if } a<a_{R} \\
0.515 \text { if } a \geq a_{R},
\end{array}\right.
$$

where $a_{R}$ is the mandatory age of retirement. The budget-constraint for Medicare is given as follows:

$$
\int_{a_{R}}^{\omega}\left[1-\phi_{M C}(a, t)\right] p_{H}(t) h(a, t) N(a, t) d a=\hat{\tau}_{M C}(t) w(t) L(t)
$$

where $1-\phi_{M C}(a, t)$ is the share of health costs paid by Medicare and $\hat{\tau}_{M C}$ the payroll tax for Medicare.

Social security, received by retirees, is financed through a payroll tax which is determined endogenously from the social security budget constraint:

\footnotetext{
${ }^{14}$ This value was calculated based on the following data of the US economy in 2003: Share of the elderly in total health spending $=40 \%$ (NHEA); health share in the GDP $=15 \%$ (NHEA); medicare share in the GDP $=2.3 \%$ (Zhao, 2014).
} 


$$
\int_{a_{R}}^{\omega} \pi(a, t) N(a, t) d a=\hat{\tau}_{\Pi}(t) w(t) L(t),
$$

where $\pi(a, t)$ is the social security pension and $\hat{\tau}_{\Pi}$ the payroll tax devoted to social security. We assume social security benefits to be exogenous and use the CPS Annual Social and Economic Supplement data for the year 2003 which states an approximately $\$ 10300$ mean social security income for individuals aged 65 years or older in 2003. Thus, we set $\pi(a, t)=\$ 10300$ for $a \geq a_{R}$ and otherwise to zero.

Altogether, individuals face the following taxes (including the premium for the private health insurance):

$$
\tau(a, t)=\underbrace{\hat{\tau}_{\Pi}(t) l(a) w(t)}_{=\tau_{\Pi}(a, t)}+\underbrace{\hat{\tau}_{M C}(t) l(a) w(t)}_{=\tau_{H}(a, t)}+\underbrace{\left[1-\phi_{P}(a, t)\right] p_{H}(t) h^{*}(a, t)}_{=\tau_{M C}(a, t)} .
$$

\section{Overview of functional forms and parameters}

Table 1 summarises the functional forms we are employing.

Table 1: Functional forms

\begin{tabular}{ll} 
Function & Description \\
\hline$u(a, t)=b+\frac{\left(c(a, t)-c_{0}\right)^{(1-\sigma)}}{1-\sigma}$ & instantaneous utility function \\
$\mu(a, t)=\widetilde{\mu}(a)\left(I(a)-\eta(a)[h(a, t) M(t)]^{\epsilon(a)}\right)$ & age-time specific mortality rate \\
$M(t) \equiv 1$ & medical technology \\
$A(t) \equiv 2.995$ & manufacturing technology \\
$B(t)=B_{0} \exp [\nu t]$ & number of births \\
$s(t)=\frac{\Upsilon_{B}(t)}{N(t)}$ & transfer from accidental inheritances \\
$Y(t)=K_{Y}(t)^{\alpha}\left(A(t) L_{Y}(t)\right)^{(1-\alpha)}$ & production in manufacturing sector \\
$F(t)=M(t)^{\gamma} K_{H}(t)^{\beta}\left(L_{H}(t)\right)^{1-\beta}$ & production in health sector \\
$\pi(a, t)=\left\{0\right.$ if $a<a_{R}, \$ 10300$ if $\left.a \geq a_{R}\right\}$ & age-specific pensions \\
$\phi_{P}(a, t)=\left\{0.51\right.$ if $a<a_{R}, 0.895$ if $\left.a \geq a_{R}\right\}$ & age-specific private coinsurance \\
$\phi_{M C}(a, t)=\left\{1\right.$ if $a<a_{R}, 0.62$ if $\left.a \geq a_{R}\right\}$ & age-specific Medicare coinsurance \\
$\phi(a, t)=\left\{0.51\right.$ if $a<a_{R}, 0.515$ if $\left.a \geq a_{R}\right\}$ & age-specific total coinsurance
\end{tabular}


The $\equiv$ symbol denotes that the function is assumed to be constant in all arguments. Table 2 summarises our choice of parameters.

Table 2: Parameters

\begin{tabular}{lll} 
Parameter & Description & Value \\
\hline$\omega$ & life span & 80 \\
$t_{0}$ & start time of optimal cohort, year 2003 & 120 \\
$\rho$ & pure rate of time preference & 0.02 \\
$b$ & constant offset for consumption in utility function & 8 \\
$c_{0}$ & subsistence minimum & $\$ 11000$ \\
$\sigma$ & inverse elasticity of intertemporal substitution & 1.75 \\
$\delta$ & rate of depreciation & 0.05 \\
$\alpha$ & elasticity of capital in $Y$ & $1 / 3$ \\
$\beta$ & elasticity of capital in $F$ & $1 / 5$ \\
$\gamma$ & efficiency of medical technology in health production & 0 \\
$\nu$ & growth rate of births & 0.013 \\
$B_{0}$ & initial number of births & 0.1 \\
$a_{R}$ & mandatory retirement age & 65
\end{tabular}

In the following, we will present the numerical results (see Appendix A4 for details on the solution of the numerical problem) for the benchmark case and three numerical experiments. We focus on a selection of the most salient outcomes. ${ }^{15}$

\subsection{Benchmark}

In order to economise on space we illustrate the benchmark allocation in the same graphs as experiment 1 (unanticipated medical advance). The benchmark allocation is depicted by blue, solid plots throughout, whereas the experiments are depicted by green, dashed plots. Some figures also contain red, dotted plots, which refer to a partial equilibrium allocation.

The salient features of the benchmark allocation can be summarised as follows. Consumption of the focal cohort, entering at $t_{0}=120$ (when they are 20 years old), is hump-shaped (see Figure 3). The fact that the interest rate (approx. 4.3\%) lies above

\footnotetext{
${ }^{15} \mathrm{~A}$ full set of outcomes is available from the authors on request.
} 
the rate of time preference $(2 \%)$ implies a rising consumption until around age 70 . Due to missing annuity markets, consumption falls, however, at higher ages as implied by the individual Euler equation (19). The demand for health care is also hump-shaped (Figure 3). While the demand for care grows very moderately up to age 40, it exhibits from then on a strong increase up to age 80 before dropping again for the highest ages. This pattern is well in line with the empirical evidence presented by Martini et al. (2007). ${ }^{16}$ The value of life (VOL) peaks at approx. age 50 (Figure 3), which is well in line with empirical evidence on the value of a statistical life in Aldy and Viscusi (2008). The remaining life expectancy at age 20 is 58.0 years in the benchmark case and, thus, matches the empirical value for the US in 2003 (58.1 years, HMD) very closely.

It is worth of note that given our assumption of constant $A, M$ and $\nu$, prices and per-capita quantities are constant in the benchmark scenario. Thus, a steady state appears to exist although we are not imposing it. The health share (in GDP) in the benchmark case is $14.4 \%$ and matches the data from the National Health Expenditure Accounts provided by CMS. ${ }^{17}$ Furthermore, the benchmark model features a Medicare share of $2.3 \%$ [2.3\% according to Zhao (2014)], a GDP per capita of $\$ 39700$ [\$39700 according to Table 1.5.5 of the revised National Income and Product Accounts of the Bureau of Economic Analysis (BEA), 2003], and health expenditures per capita of $\$ 5720$ [\$5750 according to NHEA, 2003].

Although the per capita value of health expenditure is constant, its components are subject to considerable dynamics. Consider Figure 14, which plots (in cyan, dotted) the growth rate of the per capita consumption of health care, $g_{H / N}(t)$, as well as its components, as expressed in (31). Here, the blue, solid plot refers to the growth rate of the individual consumption of care averaged across all age groups and the green (dashed) and red (dashed-dotted) plots refer to the growth impact of the entry of new born and deceasing cohorts, respectively. Focusing for the moment only on the steady state part of the plots at the beginning of the time line, we see that the stationary level of per capita consumption of health care is the outcome of two offsetting tendencies: The growth of individual consumption at a rate of $3.2 \%$ is offset by the drag on per capita consumption through incoming cohorts with below average consumption $(-2.1 \%)$ and through the death of individuals with above average consumption $(-1.2 \%)$.

\footnotetext{
${ }^{16}$ Note that this pattern is not inconsistent with the finding that health care utilisation/expenditure increases with the closeness to death (e.g. Zweifel et al. 1999). This is because the "cost of dying" itself is declining with age for the highest ages (e.g. Cutler 2007).

${ }^{17}$ The numerator of the GDP health share is the total health expenditure $p_{H}(t) \cdot H(t)$ and the denominator is the $G D P(t)=p_{H}(t) \cdot H(t)+Y(t)$.
} 
The exogenous number of births and endogenous death toll result in a stable population growth of $1.31 \%$, which differs from the $0.9 \%$ growth rate the US experienced in 2003. However, we do not intend to match the population growth rate but rather the share of the elderly population in the year 2003. Moreover, our implied growth rate matches the trend observed between 1920-2000 (US Census, 2000).

Before setting out on the experiments a clarifying remark is warranted on the purpose and design of our numerical analysis. The main objective of our analysis lies in an analytical and quantitative understanding of the mechanisms which are underlying the macro-economic impacts of medical and demographic change. In order to avoid that these impacts are confounded by other sources of change, we have structured our numerical analysis in a way that the economy is "quasi-stationary" in the years surrounding the shock. This is why we are abstracting from time-trends in the states of technology, $A(t)$ and $M(t)$ as well as in the birth rate $\nu$, the appropriate calibration of which would, of course, allow us to arrive at a more realistic dynamic representation of the economy. ${ }^{18}$ This notwithstanding, we have calibrated the model to the US economy in the year 2003 in order to provide a realistic static backdrop for our experiments.

\subsection{Experiment 1: Unanticipated Medical Advance}

We consider here an unanticipated increase in the state of the medical technology from $M(t)=1$ for $t \leq 150$ to $M(t)=2$ for $t>150 .{ }^{19}$ The timing implies that the focal cohort, entering the model at $t_{0}=120$, is aged 50 at the point of the innovation. We assume that the medical technology does not bear on the production of health care,

\footnotetext{
${ }^{18}$ For instance, we could match both, the age-structure and the rate of population growth in 2003 by assuming an appropriate time-profile of the birth rate $\nu$ prior to the year 2003 . While this would give us a (more) realistic description of the demographic change following the year 2003, the impact of this on the economy would interfere with our experiments.

${ }^{19}$ To gauge the magnitude of the medical innovation, we consider a 50 year old individual. Considering the steady-state values, we find that the innovation raises the remaining life-expectancy of a 50 year old by some 1.1 years and induces additional (discounted) expenditures of about $\$ 19000$ over the remaining life-course. These magnitudes are not grossly out of line with evidence provided by Cutler (2007) on the impact of revascularisation, as was introduced into the US during the late 1980s. Cutler finds that for a patient with myocardial infarction, revascularisation would raise life-expectancy by about 1 year and induce about $\$ 40000$ in additional expenditure. While the impact of innovation in our model is, thus, comparable in the order of magnitude, it should be borne in mind that the figures are not directly comparable, as in Cutler the values apply (ex-post) to individuals who have had a heart attack, whereas in our model they apply (ex-ante) to a representative agent.
} 
its only effect is to render more effective the use of health care in lowering mortality ${ }^{20}$. While the absence of a supply-side impact of the innovation may be unrealistic, this allows us to isolate demand-driven general equilibrium effects arising from medical innovations.
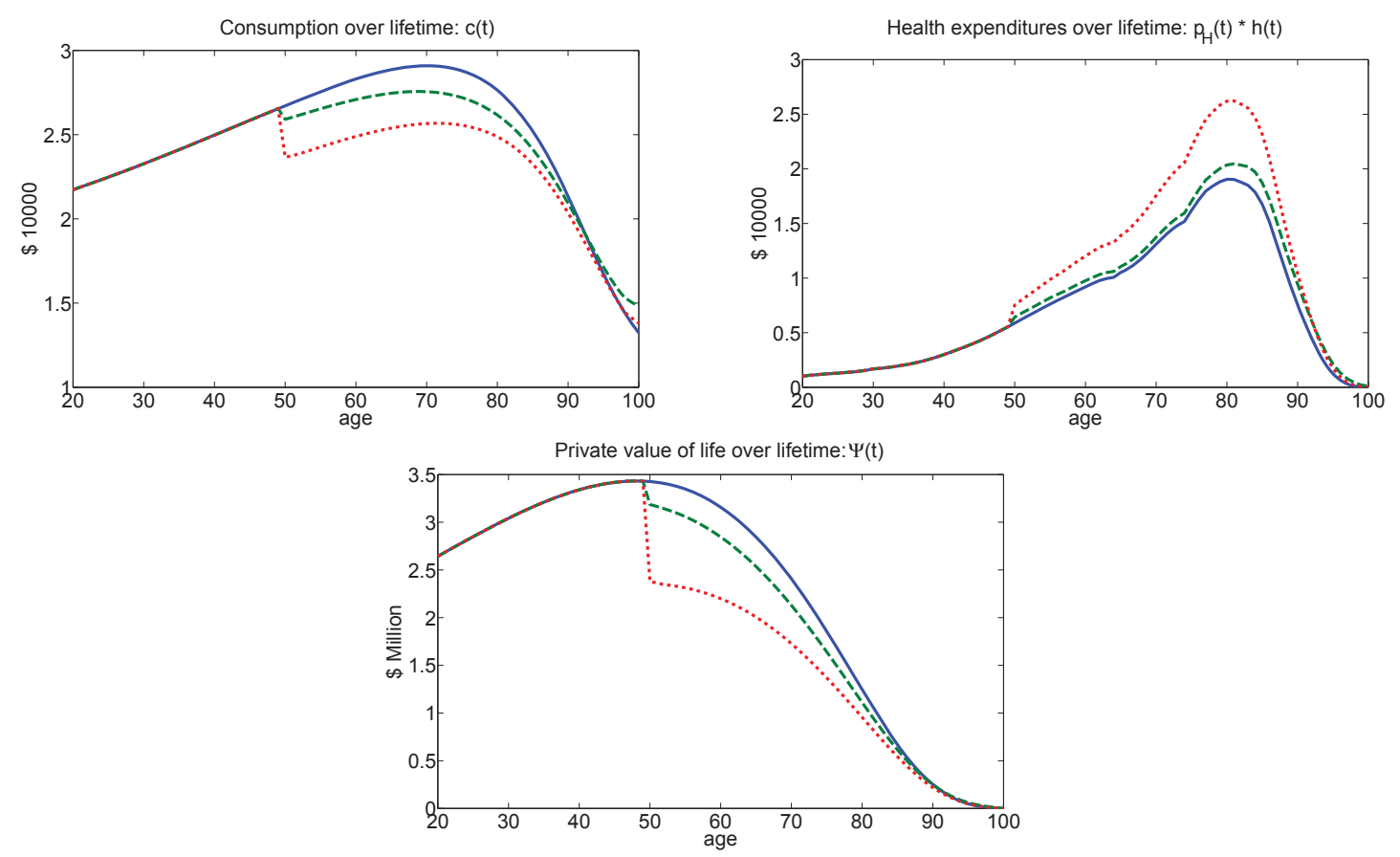

Figure 3: Life-course consumption, health expenditure and value of life profiles for benchmark case (blue, solid line), for the unanticipated shock of $M$ in the general equilibrium (green, dashed line) and the partial equilibrium effect (red, dotted line)

At the level of the individual, we find the following effects of an unanticipated medical advance: As Figure 3 illustrates, and as one would expect, the innovation induces individuals at age 50 to reallocate expenditure from consumption to health care. Indeed, the drop in consumption is persistent over the remaining life-cycle. When it comes to the impact of the innovation on the demand for health care (as measured by individual health expenditure), a more ambiguous picture emerges in Figure 3: For a given set of prices, the expenses for medical care would increase for all age groups by a substantive amount (see the red, dotted plot). However, such a partial equilibrium take

\footnotetext{
${ }^{20}$ To see this note that

$$
\begin{aligned}
\mu_{h}(a, t) & =-\widetilde{\mu}(a) \eta(a) \epsilon(a) M(t)^{\epsilon(a)} h(a, t)^{\epsilon(a)-1}<0, \\
\mu_{M}(a, t) & =-\widetilde{\mu}(a) \eta(a) \epsilon(a) M(t)^{\epsilon(a)-1} h(a, t)^{\epsilon(a)}<0, \\
\mu_{h M}(a, t) & =-\widetilde{\mu}(a) \eta(a)(\epsilon(a))^{2}[M(t) h(a, t)]^{\epsilon(a)-1}<0 .
\end{aligned}
$$
}


is inappropriate, as the general equilibrium impact of the innovation on the underlying demand and supply system needs to be taken into account. Once we do this, much of the demand expansion vanishes (see green, dashed plot). While individual health expenditures increase over the benchmark level by a small amount between ages 50 and 80, they barely rise above the benchmark for the highest ages. This notwithstanding, the medical innovation raises remaining life-expectancy at age 20 from 58.0 to 59.1 for a member of the focal cohort. Notably, the strong increase in demand for a constant set of prices would induce an additional gain of only 0.35 life years.
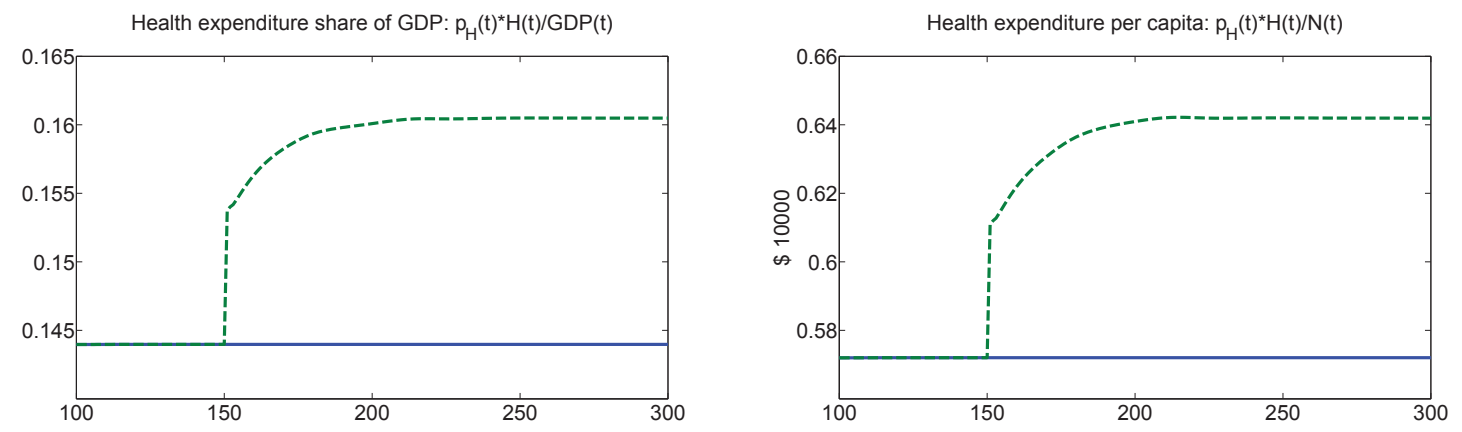

Figure 4: Macroeconomic variables

The innovation at $t=150$ induces a sudden and permanent increase in the per capita demand for health care, which is mirrored by the increase in the health expenditure share of the GDP, $p_{H}(t) H(t) / G D P(t)$, by some 1.7 percentage points (Figure 4 ). The shift from final goods production to health care that is ensuing the innovation leads to a reduction of the employment share in the manufacturing sector, a reduction in the interest rate and an increase in the wage rate (see Figure 5). The change in the factor prices comes with an increase in the price of health care, which is underlying the dampening of the demand increase. Furthermore, the social security payroll tax rises, following the pronounced increase in longevity, despite the simultaneous increase in the gross wage. Similarly, Medicare payroll taxes increase as a consequence of both greater health spending and the boost in longevity. These sectoral and price adjustments notwithstanding, the medical advance has very little impact on GDP per capita (not shown graphically). The survival gains induced by the innovation are greatest among older cohorts and, for a fixed retirement age, lead to a reduction in the employmentpopulation ratio by about 1 percent. ${ }^{21}$ At the same time, however, the expansion of

\footnotetext{
${ }^{21}$ The medical innovation raises the remaining life expectancy at age 20 by 1.0 years from 58.04 years (and, thus, by 1.3 percent) and remaining life expectancy at age 65 by .81 years from 18.02 years (and, thus, by 4.5 percent).
} 

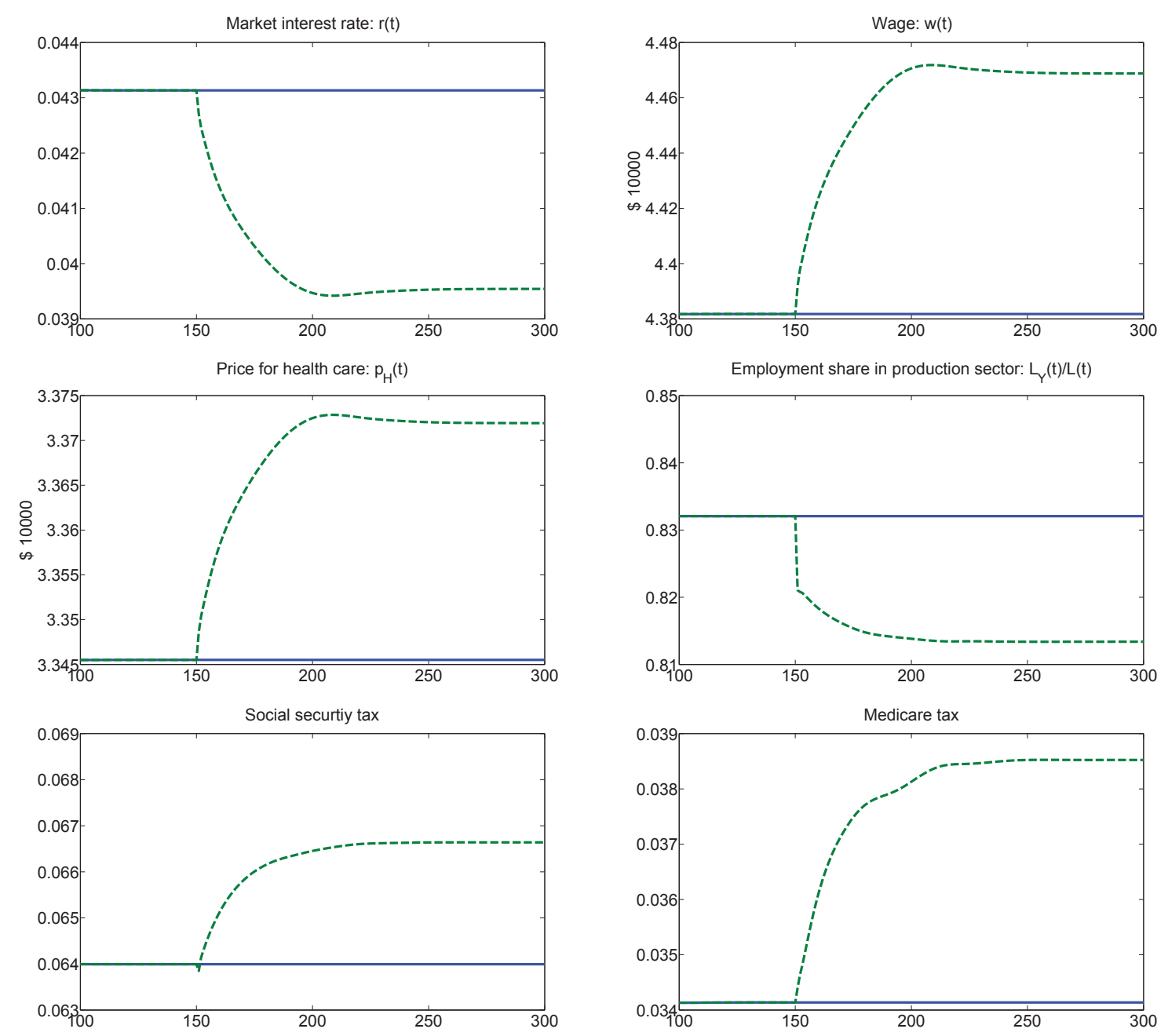

Figure 5: Market prices, employment share and taxes

the expected retirement period triggers additional savings, translating into an increase in the capital stock per capita. Overall, this shift towards a (mildly) more capital intensive economy is balancing out in its impact on GDP per capita.

Although per capita demand for health care and the associated expenditure, $p_{H}(t) H(t) / N(t)$, have increased after the innovation, the magnitude of the effect varies across age-groups. Specifically, those over 80 exhibit a very modest demand increase in spite of the innovation. For these cohorts the willingness to pay for care, as measured by the VOL, is so low that the value of the survival gains from the innovation barely outweighs the price increase. Finally, and strikingly, the medical innovation leads to a reduction in the VOL of the focal individual past age 50 at which the innovation has become available (see Figure 3). At face value, the lower willingness to pay for survival 
follows from the reduction in consumption over the remaining life-course. ${ }^{22}$

However, a different interpretation can be attached to it in light of the fact that the demand of health care is non-decreasing in response to the medical innovation over the full life-cycle. Rewriting the first-order condition for the demand of health care (16) to $\psi(a, t)=-\phi(a, t) p_{H}(t) \mu_{h}^{-1}$, we find that the VOL is equated to the effective (or quality-adjusted) price of medical care $-\phi(a, t) p_{H}(t) \mu_{h}^{-1}$, the latter depending on both the market price and the marginal impact on mortality of health care, $-\mu_{h}$. Recalling that $\mu_{h h}>0$, an increasing demand for care would ceteris paribus imply a greater effective price. But then it must be true that the medical innovation has lowered the effective price for medical care (recall that $\mu_{h M}<0$ ) to an extent that it over-compensates the increase in the market price, $p_{H}(t)$. Notably this finding is consistent with evidence produced by Cutler et al. (1998) who find that while the price for heart attack treatments, as measured by a Service Price Index, was increasing over the time span 1983-1994, the quality-adjusted price was effectively declining. We can thus summarise a first set of insights.

Result 1 (i) The general equilibrium impact of a mortality reducing medical innovation on the demand for health care tends to be dampened by an associated price increase. (ii) The positive effect of medical innovation may differ in magnitude across cohorts and is very modest for age-groups with a low VOL. (iii) Medical innovation tends to raise the capital intensity of the economy throughout, with little impact on GDP per capita. (iv) Medical innovation leads to a reduction in the VOL and in the effective (quality-adjusted) price for medical care.

\subsection{Experiment 2: Anticipated Medical Advance}

In many instances, medical advances do not arrive as "shocks", but they are anticipated in terms of prior medical research and/or the clinical trials leading to the admission of new medical technologies or pharmaceuticals. Thus, it is appropriate to take into account consumers' anticipation of such innovations. In the following, we consider once again a medical innovation from $M(t)=1$ to $M(t)=2$, but assume now that it is fully anticipated. In order to gain a better understanding of the anticipation effect we assume that the innovation is taking place at $t=200$, with the focal cohort being entering at $t_{0}=170$.

\footnotetext{
${ }^{22} \mathrm{~A}$ decomposition of the change in the VOL in response to the medical innovation shows that for a (counterfactually) fixed consumption profile, the increase in remaining life-expectancy following the innovation would increase the VOL. This impact is, however, vastly over-compensated by the decline in consumption, following the reallocation of expenditure toward health care.
} 

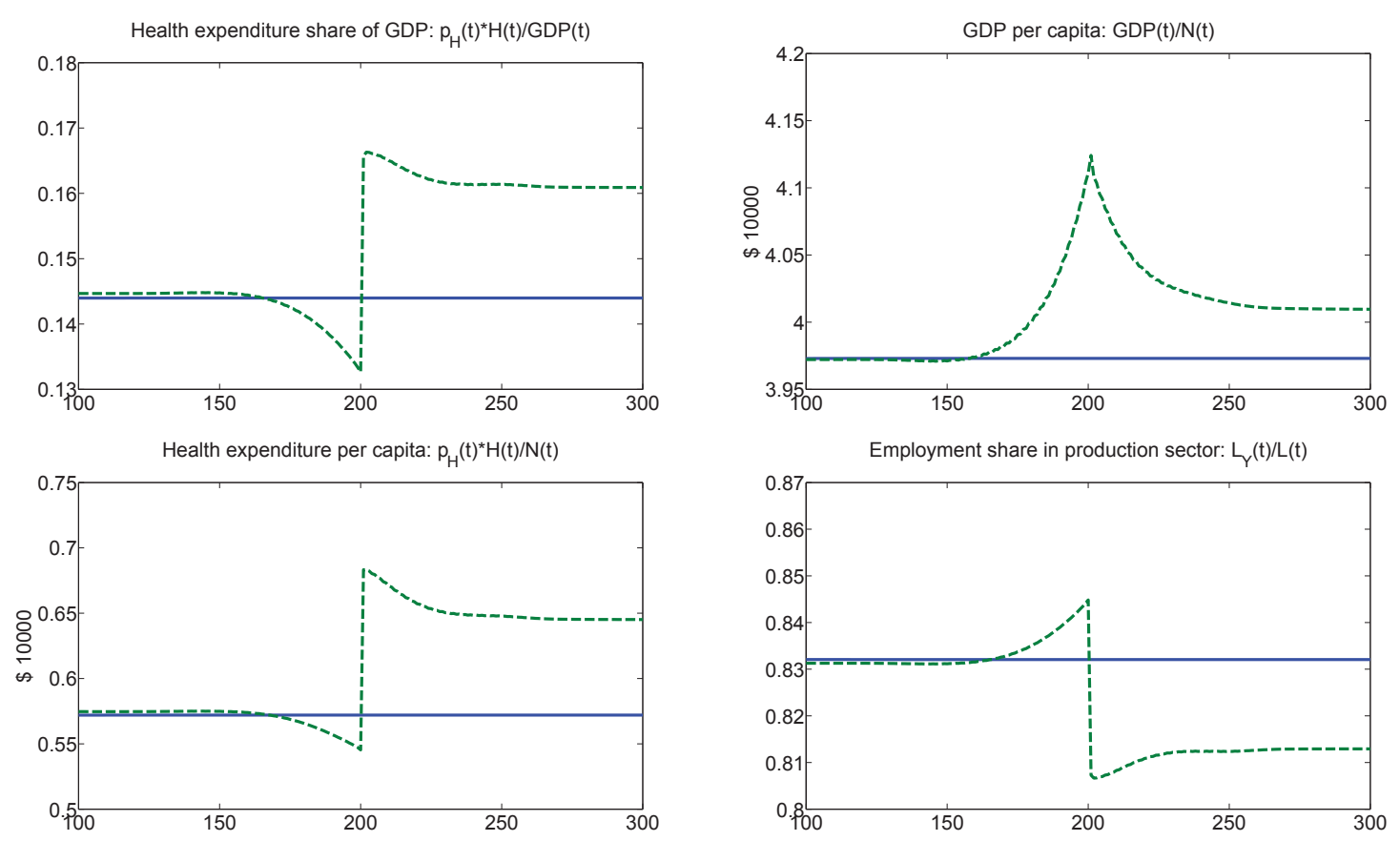

Figure 6: Macroeconomic variables

In order to appreciate the role of anticipation it is instructive to consider first the macro-economic impact of the innovation. Figure 6 plots how the health share of GDP, the health care expenditures per capita, and the employment share in the production sector, $L_{Y}(t) / L(t)$ respectively, develop over time. Each of the three quantities exhibits a particular pattern, reflecting the impact of anticipation at aggregate level. Reading the figures backwards in time, the innovation at $t=200$ eventually leads to the expected increase in the health share and in the per capita expenses on health care over and above their respective benchmark levels, as well as to a corresponding shift of employment from production to the health care sector. ${ }^{23}$

Notably, however, for a time span of about 30 years before the innovation, health expenditures (and consequently the health share) fall below their benchmark levels. This amounts to an anticipation effect, where individuals postpone the consumption of care to wait for the innovation to occur. ${ }^{24}$ The corresponding shrinking of the health care sector is reflected in a temporary boost to the employment share in final goods

\footnotetext{
${ }^{23}$ GDP per capita exceeds the benchmark level by a small amount, reflecting the steady-state increase in financial wealth and the capital stock due to higher longevity after the innovation.

${ }^{24}$ Such a demand-reducing anticipation effect has been identified in regard to the consumption of pharmaceuticals prior to the Medicare D reform aimed at including pharmaceutical expenditure into the coverage (Hu et al. 2014; Alpert 2015).
} 
production. ${ }^{25}$
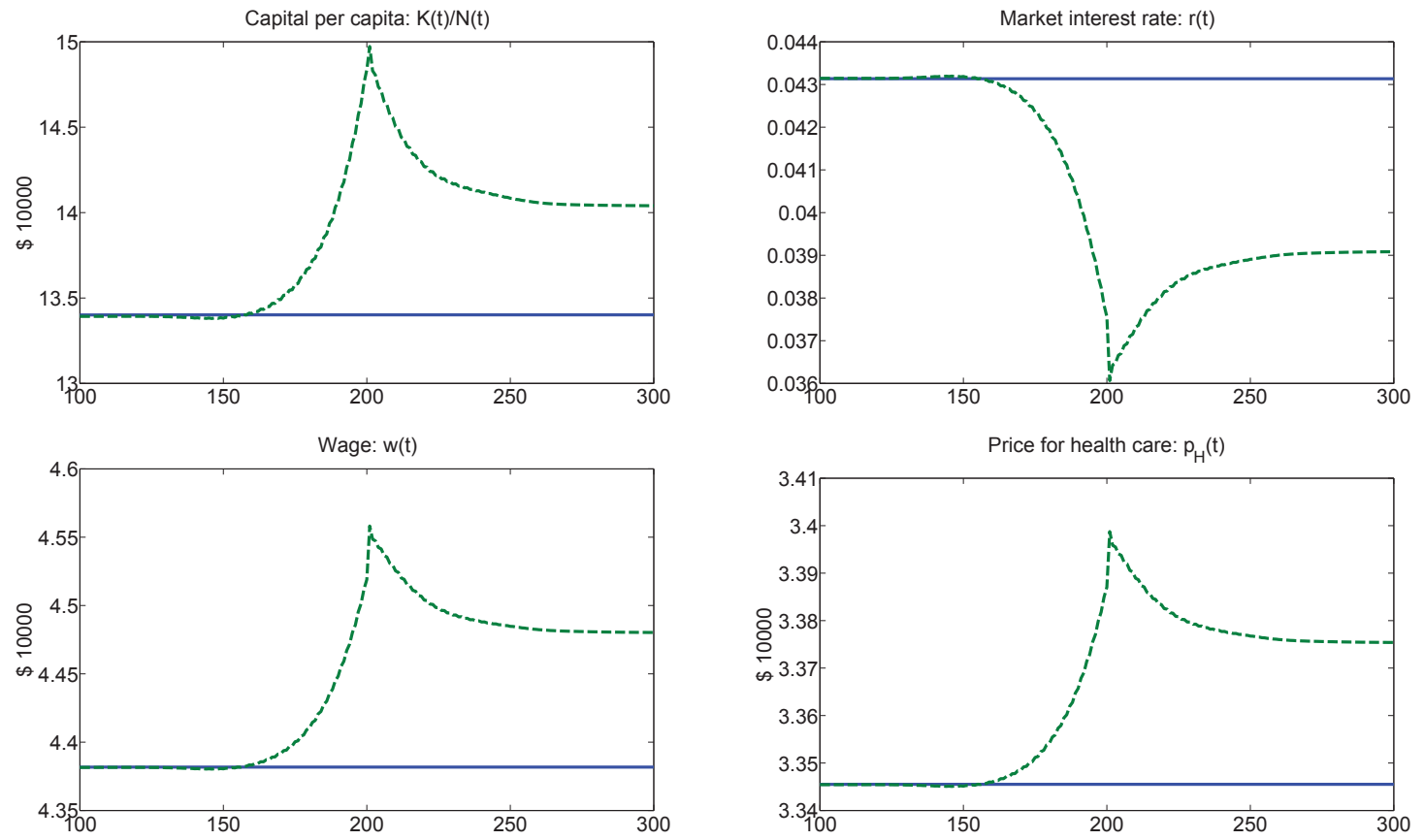

Figure 7: Capital per capita and market prices

Figure 7 plots the development of the capital per capita, $K(t) / N(t)$, the market interest rate, $r(t)$, the wage rate, $w(t)$ and the price for health care, $p_{H}(t)$. The paths show a pattern that differs distinctly from the one arising in the case of an unanticipated shock (recall Figure 5). The postponement of health expenditures over the anticipation period translates into higher saving, an effect that is complemented by an ancticipative reduction in per capita consumption below its benchmark (not shown here). ${ }^{26}$ The resulting boost to the capital held by individuals triggers a decline in the interest rate and a boost to the wage rate. With the health care sector being relatively labour intensive, the increase in the wage rate drives up the price for health care despite

\footnotetext{
${ }^{25} \mathrm{~A}$ close-up look shows that the anticipation-related slump in the demand for health care itself is, in turn, anticipated in as far as prior to the slump, the demand for health care and the employment share in health care are slightly elevated over and above their benchmark levels. Overall, this amounts to an anticipation wave, akin to the one described by Feichtinger et al. (2006) for the impact of technological progress on capital accumulation.

${ }^{26}$ One could argue that the reduction in health care in anticipation of an innovation lacks realism in as far as health care bears on survival. We certainly do not wish to imply that individuals facing lifethreatening conditions are deferring treatments. However, anticipatory adjustments are quite probable in regard to the intensity of given treatments such as e.g. drug prescriptions (Alpert 2015; Kaplan and Zhang 2016). They are also conceivable in as far as the utilisation of distinct treatments with different intensities respond to current and expected prices and benefits (e.g. Cutler and Huckman 2003 for treatments of coronary disease). For our representative consumer approach, changes in the distribution of treatments across the patient population translate into marginal adjustments in the intensity of care.
} 
the deferral of demand. At the arrival of the medical innovation, individuals begin to dissave in order to purchase greater quantities of what is more effective health care now, and over time capital per capita falls back to its new "quasi steady-state" level, which as with the non-anticipated advance lies somewhat above the benchmark. The factor prices and the price for health care do not return to their initial levels either. The reason for this lies with the post-innovation shift of economic activity towards the more labour intensive health care sector. Hence, while prices are driven by the supplyside over the anticipation period, they tend to be determined by the demand-side after the innovation. Finally, the boost in capital per capita over the anticipation period translates into a temporary boom of the economy, as measured by GDP per capita (see Figure 6).

As compared to the previous case of a non-anticipated medical innovation, anticipation does not vastly alter the life-cycle allocation of the focal cohort. ${ }^{27}$ One distinction is that consumption is reduced smoothly over the full life-cycle, allowing the individual to avoid the utility loss from a sudden drop in consumption at the arrival of the innovation. ${ }^{28} \mathrm{~A}$ comparison of the wealth profiles across the cases with and without anticipation shows that some of the early-life consumption foregone by an anticipating individual is, indeed, used for the accumulation of extra savings for the later purchase of health care. As Figure 8 shows the smoothing of consumption has a noticeable impact on the value of life for the focal cohort, which in the absence of anticipation exhibits a sharp drop at the age of 50 (the age at which consumption is reduced sharply) only to be recuperated afterwards. Under consumption smoothing this drop is avoided. While this necessarily implies a crossing of the VOL at the point of innovation, reflecting the crossing of consumption schedules, the equalisation of the VOL with the effective price of medical care lends itself to an interesting observation. When anticipated, the innovation leads to a less pronounced fall in the effective price of medical care. This is consistent with the fact that the nominal price for medical care peaks at the point of innovation (see Figure 7). With the price peak fading away over the years, the steady-state impact of medical progress on the effective price of medical care is more pronounced. Whenever anticipation effects are relevant, the closeness to innovation (in terms of time), therefore, needs to be taken into account in estimations of the impact of innovation on medical prices (as well as on other outcomes).

\footnotetext{
${ }^{27}$ Likewise, the remaining life expectancy at age 20 increases only by an additional 0.05 years.

${ }^{28}$ Interestingly, the strong fall in the interest rate in the years leading up to the innovation does not translate into a sizeable shift of the consumption profile of the focal cohort. This may be due to the relatively low intertemporal elasticity of substitution of $\sigma^{-1}=4 / 7$.
} 


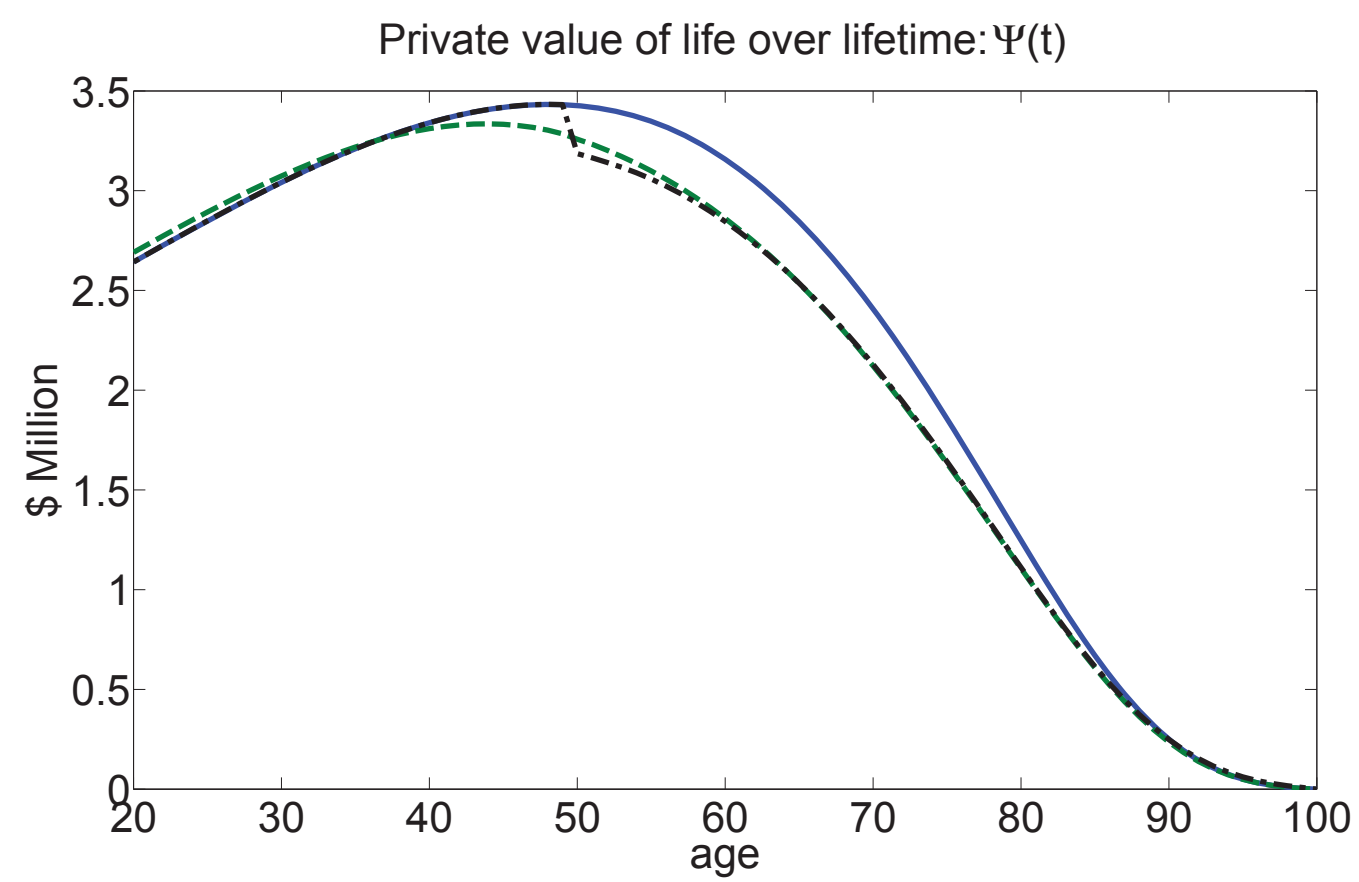

Figure 8: Private Value of life for the benchmark case (blue, solid line), for the anticipated shock of $M$ (green, dashed line) and for the unanticipated shock of $M$ (black, dashed-dotted line)

We conclude this experiment by isolating the drivers behind the changes in the level of per capita health expenditure. Figure 9 a decomposes the change in health expenditure from the benchmark (blue, solid line) to the outcome under the anticipated medical advance (cyan, dotted line) into two partial effects: a price effect (red, dasheddotted line), holding constant per capita demand $H(t) / N(t)$ at the benchmark level; and a demand effect (green, dashed line), keeping the price at the benchmark level. While the price increase is dampening the decline in per capita health expenditure in anticipation of the innovation, and while its subsequent persistence at a higher level is magnifying the increase in per capita health expenditure after the innovation, the overall impact of the price change is relatively small, accounting for roughly $8 \%$ of the overall increase in per capita expenditure at the point of innovation. Figure $9 \mathrm{~b}$ decomposes the changes in the per capita demand for health care (blue, solid line $=$ baseline; cyan, dotted line $=$ experiment) into a component that reflects changes in the levels of individual demand, $h(a, t)$, for the baseline age-structure of the population (red, dashed-dotted line); and a component that reflects changes in the age-structure for the baseline age-profile of individual demand (green, dashed line). As one would expect a substantial impact through a shift in the age-structure sets in only after the medical advance. Notably, the shift towards an older population explains about $15 \%$ 
of the steady-state increase in per capita health expenditure. Thus, the shift in the age-structure dampens to considerable extent the reduction in the per capita demand for health care, following its peak at the point of innovation. ${ }^{29}$

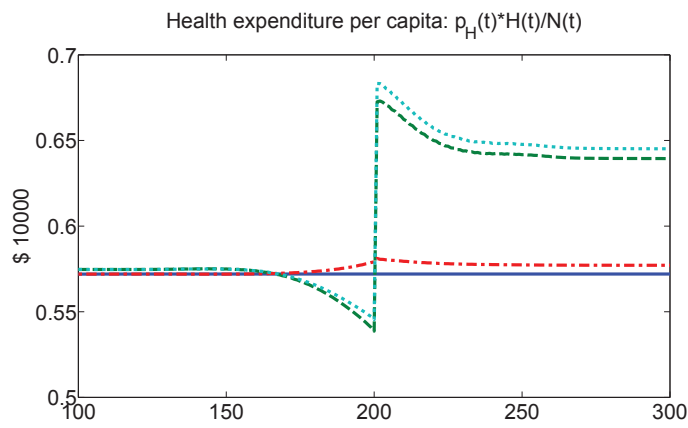

(a) Benchmark (blue, solid) Anticipated med. advance (cyan, dotted) Benchmark $h(a, t)$ (red, dashed-dotted) Benchmark $p_{H}(t)$ (green, dashed)

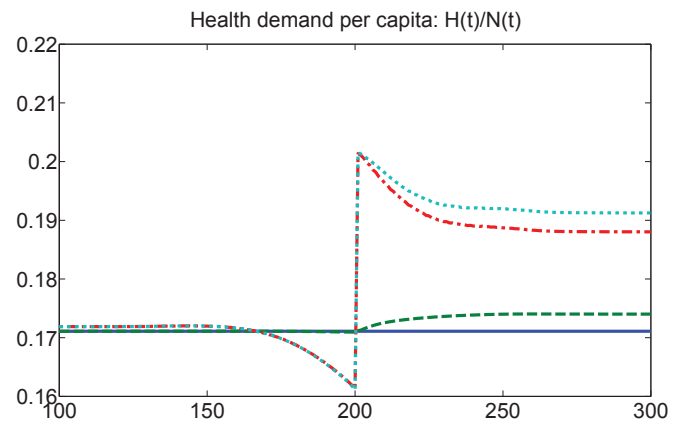

(b) Benchmark (blue, solid) Anticipated med. advance (cyan, dotted) Benchmark $N(a, t)$ (red, dashed-dotted) Benchmark $h(a, t)$ (green, dashed)

Figure 9: Decomposition of per capita health expenditures and demand

We can summarise as follows.

Result 2 The anticipation of a mortality reducing innovation leads to (i) the contraction of the demand and supply for health care to a level below the benchmark for a period prior to the innovation; (ii) the accumulation of extra capital prior to the innovation and for a certain period, following the innovation; and (iii) to a concomitant reduction (increase) in the interest rate (wage rate and price for health care) prior to the innovation. (iv) By inducing extra saving, anticipation generates a temporary economic boom. Individuals from the focal cohort, (v) lower consumption in a smooth way over the whole life-cycle, allowing them to sustain a higher value of life relative to the case without anticipation. (vi) The changes in health expenditure per capita before and after an anticipated innovation are predominantly demand driven rather than price driven, with a shift towards an older population explaining about $15 \%$ of the steady-state increase in demand.

\subsection{Experiment 3: Baby Boom}

As a third experiment, we consider the impact of an unanticipated baby boom. Specifically, we assume that over the time interval $t \in[151,160]$ the number of births doubles as compared to the benchmark.

\footnotetext{
${ }^{29} \mathrm{~A}$ similar decomposition for the case of an unanticipated shock shows that the impacts are similar to the post-innovation impacts depicted in Figures 9a and $9 \mathrm{~b}$.
} 

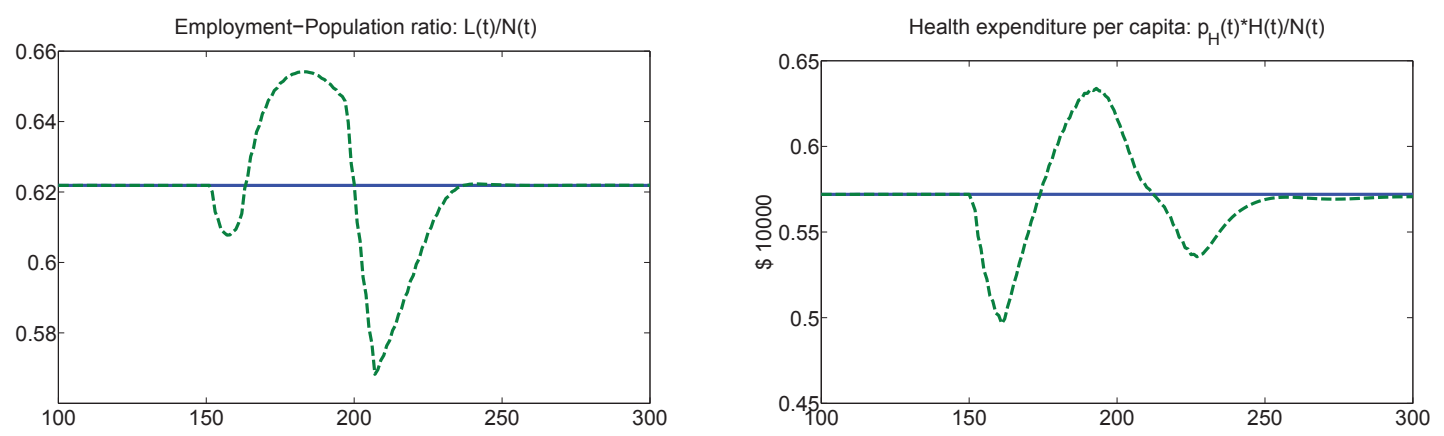

Figure 10: Employment-population ratio and health expenditures per capita for the benchmark case (blue, solid line) and the baby boom (green, dashed lined)

The baby boom is reflected in the employment-population ratio, as depicted in Figure 10. While the baby boom translates into a strong increase in the effective supply of labour per capita over the first 50 years or so, this boom is followed by a bust once the majority of the baby-boomers have retired and need to be supported by the smaller working generations. ${ }^{30}$ After the last baby boomers have deceased, effective labour supply per capita returns to its benchmark value. GDP per capita follows the same pattern (not shown graphically). As the second panel in Figure 10 illustrates, the movement of the baby boom cohorts through their working lives and subsequent retirement entails a distinct development over time of the health expenditures per capita. Health expenditures per capita fall as large cohorts of young individuals with a low demand for care enter the population during the baby boom. Afterwards, health expenditures per capita begin to grow, and they do so under the influence of two forces: (a) As Figure 11 shows, the entry of the baby-boomers into the labour market leads to a temporary reduction in the wage rate, an effect that is reversed when the baby-boomers enter retirement. The availability of additional resources for the labourintensive health care sector triggers a reduction in the price for health care, which in turn induces a supply-driven health care "boom", benefiting especially the older generations at the time. (b) With the subsequent ageing of the baby-boom cohorts into life-years with a high demand for health care, the increase in health expenditures per capita is predominantly demand-driven. ${ }^{31}$ While blurring into each other in regard to their impact on health expenditure per capita, the supply-side and demand-side forces

\footnotetext{
${ }^{30}$ The small initial drop in the employment-populatin ratio is owing to the relatively low employment rates of the very young baby-boomers.

${ }^{31}$ Notably, there is a reversal of health expenditure per capita, dropping below the benchmark, and of the various prices during the final stages of the baby boom (approximately $t=220-250$ ). We will comment on this further on below.
} 
induce a twin peak of the Medicare tax, $\hat{\tau}_{M C}$, in Figure 11. The Medicare tax peaks first at approx. $t=180$, caused by the supply-driven boom of health expenditures among the elderly, but falls subsequently until $t=200$, the years in between being those in which the economy still benefits from the large baby boomer cohorts. As soon as the baby boomers enter retirement around $t=200$, however, the demand-driven boom of health expenditures per capita leads to a drastic increase in the Medicare tax, which falls back to the benchmark level only as the baby boom cohorts decease. Although not presenting this graphically, we find that the social security tax broadly follows the expected pattern: for the defined benefit pay-as-you-go pension system we are assuming, the baby boom allows an initial reduction in the social security tax. This is sharply reversed, however, from the year $t=200$, where the high dependency ratio implied by the retired baby-boom generations calls for higher contributions up to the point the baby boomers have left the economy.
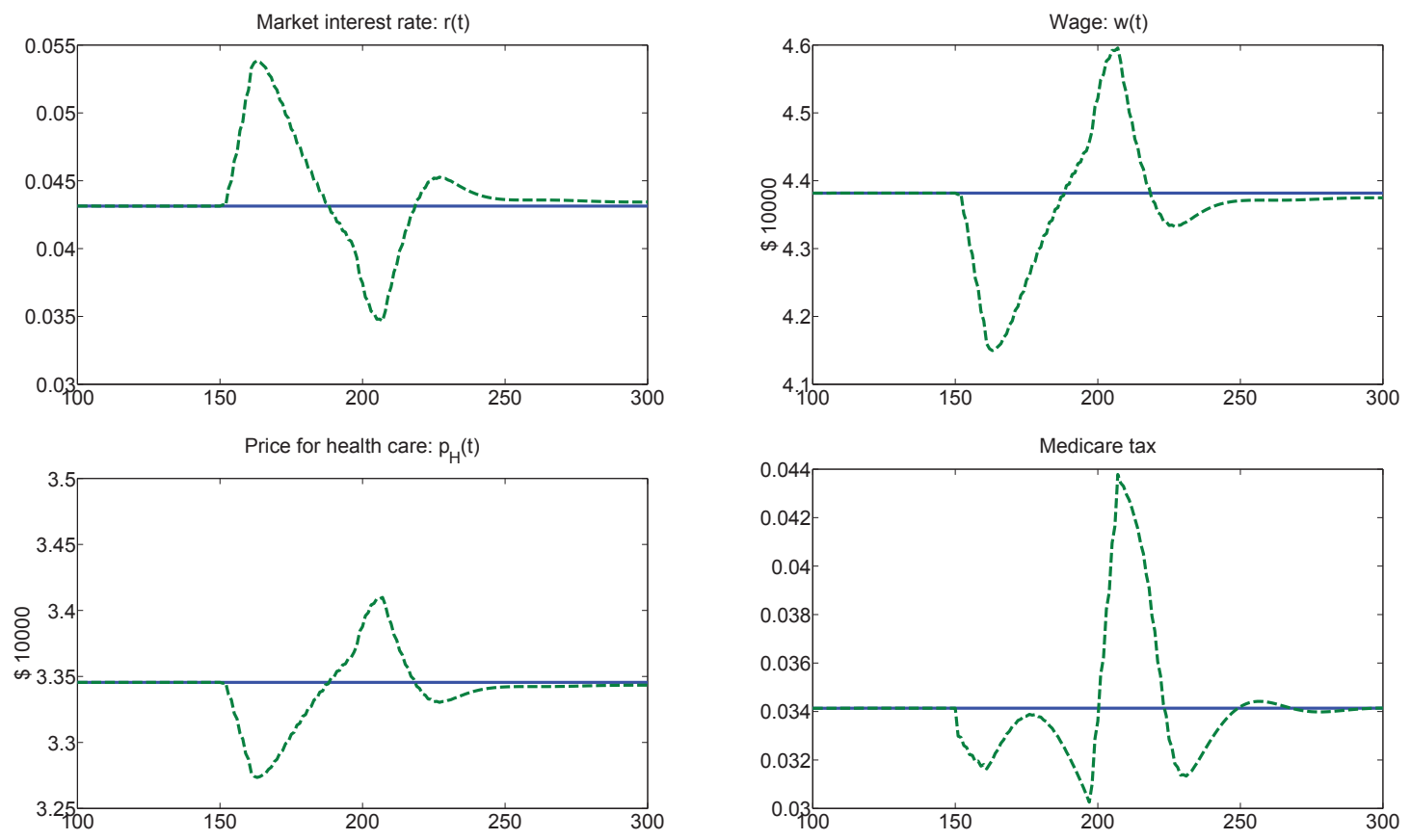

Figure 11: Interest rate, wage, price for health care, and the Medicare pay roll tax, $\hat{\tau}_{M C}$

Figure 12 illustrates the impact of the baby-boom on the life-cycle allocation of two distinct cohorts in relation to the benchmark cohort. Note that the figure now has age on the horizontal axis, with the two experimental cohorts being indexed by the entry year $t_{0}=\{100,150\}$. Given the quasi steady-state in the benchmark scenario, it is immaterial which particular entry year in the range $[100,150]$ we assign to the 
benchmark cohort. Clearly, individuals from the early born cohort $\left(t_{0}=100\right)$ benefit from the baby boom that sets in when they are aged $70 .{ }^{32}$ This is manifest in a higher level of both consumption and health care expenditure. With health care bought at a lower price, the increase in health care expenditure indicates an even stronger increase in the consumption of care. The increase in the interest rate above its benchmark during the labour boom implies that both consumption and health care are shifted into higher ages. In contrast, the (first) baby boom cohort $\left(t_{0}=150\right)$ sustains sizeably lower levels of consumption and health care from their 70 s onwards. Interestingly, however, members of this cohort exhibit a slightly higher level of consumption and health care over the age range 40-70. The increase in consumption reflects the sharp decline in the interest rate the cohort experiences during this phase of their life-course, which coincides with the time period at which the labour boom is fading. Over the same time interval, the baby boom cohort seeks to advance health care expenditure in anticipation of the high prices of care they expect for their later years.

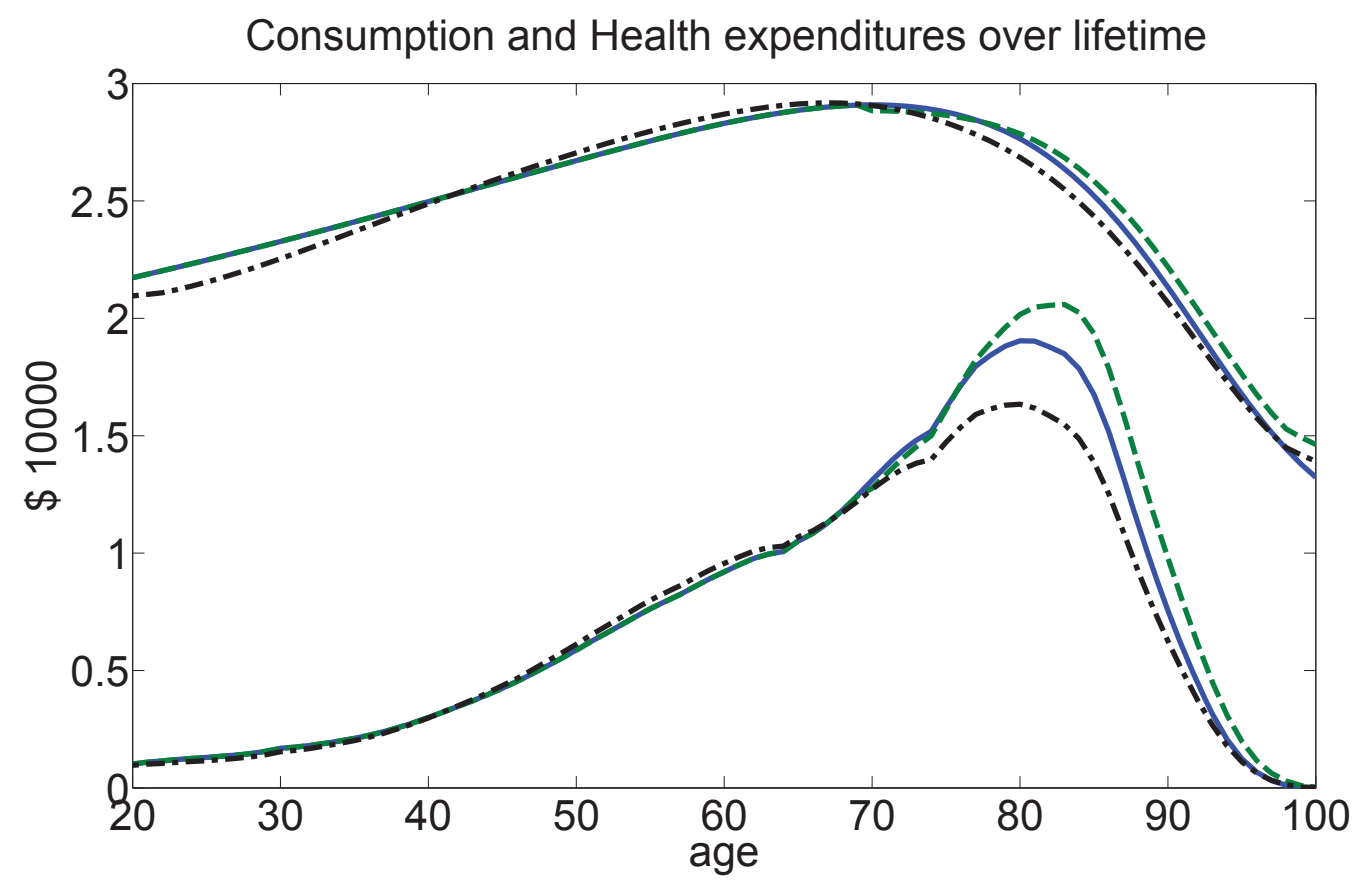

Figure 12: Consumption (upper plots) and health expenditures (lower plots) for the benchmark case (blue, solid line), the baby boom scenario with cohort born at $t_{0}=100$ (green, dashed lined) and cohort born at $t_{0}=150$ (black, dashed-dotted line)

Again, it is instructive to decompose the changes in the per capita level of health expenditure. Figure 13a shows that, while having the expected direction, the pure price

\footnotetext{
${ }^{32}$ Notably, the cohort born at $t_{0}=100$ is able to take full advantage of the baby boom in as far as it is already in retirement at the point at which the labour boom leads to a depression of earnings.
} 
movement (red, dashed-dotted line) explains only a small share of the total development of per capita health expenditures in response to the baby boom. As Figure 13b shows, the development of the per capita consumption of health care, $H(t) / N(t)$ in response to the baby boom (cyan, dotted line) is driven by two strong countervailing movements. For individual demand fixed to its age-specific benchmark levels, the development of the age-structure in response to the baby-boom leads to the expected decline in the percapita consumption of health care while the baby-boomers are in their youth, followed by an increase above the baseline once they are old (green, dashed plot). Notably, the changing age-structure does not explain the drop in health expenditure per capita during the final stages of the baby boom (approximately $t=220-250$ ). In this respect, we need to refer to the impact of the baby boom on the individual demand for health care, given the benchmark age-structure of the population (red, dashed-dotted line). We have hinted already at the increase in the individual demand for health care during the labour market boom. Initially, this effect is more than offset by the very young age-structure, implying the early dip in the per capita demand for health care. With the ageing of the baby boomers, however, the increase in health care demand becomes increasingly dominant, and the peak in the per capita consumption of health care is, indeed, explained both by the baby-boomers having shifted into age-groups with above average consumption and by the ongoing boost of individual demand levels over the baseline. As we have seen earlier, the baby boom cohorts themselves and later born cohorts tend to experience substantive reductions in their net life-cycle income due to the high price for health care and high tax rates during the years at which the baby boomers are retired. Figure $13 \mathrm{~b}$ reveals the strong extent to which the ensuing reduction in the demand for health care depresses the per capita demand of health care over the late phase of the baby boom, reaching well into periods in which most of the baby-boomers have deceased.

We round off the analysis by studying how the baby boom bears on the growth of the per capita consumption of health care as well as on its components. The growth rates of per capita health care depicted in the cyan (dotted) line in Figure 14 explain the movement of the level of per capita health care, as discussed before. Considering the components of growth, it emerges that, unsurprisingly, the initial sharp drop in per capita consumption is entirely driven by the strong increase in birth rates (green, dashed line). Interestingly, the sudden reduction in the birth rate at the end of the baby boom (after ten years) leads to a reversal of this effect and, thus, to a "snapping back" of the growth rate, for a number of years. The baby boom is accompanied by 


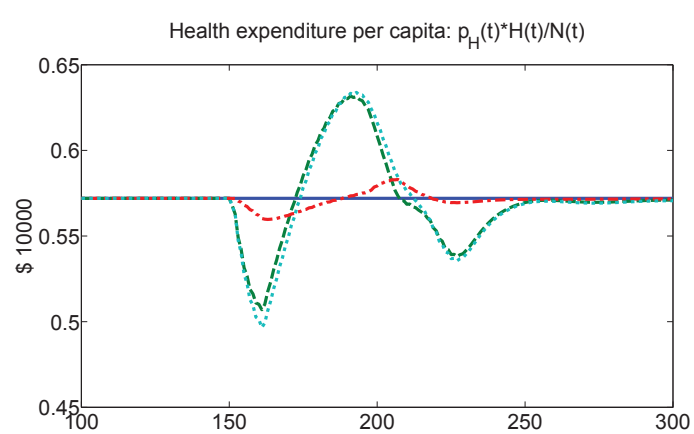

(a) Benchmark (blue, solid)

Baby boom (cyan, dotted)

Benchmark $h(a, t)$ (red, dashed-dotted)

Benchmark $p_{H}(t)$ (green, dashed)

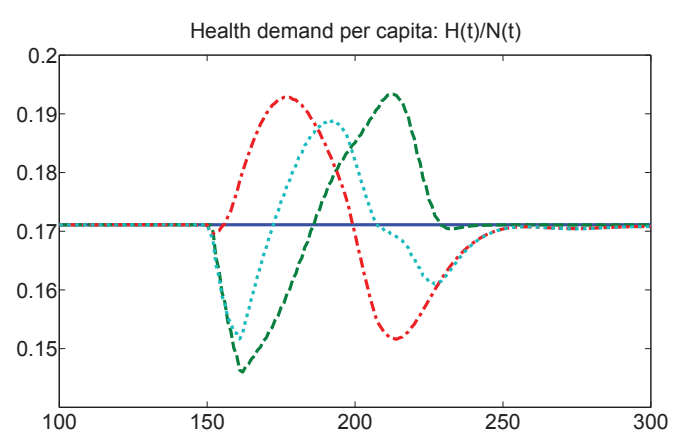

(b) Benchmark (blue, solid)

Baby boom (cyan, dotted)

Benchmark $N(a, t)$ (red, dashed-dotted)

Benchmark $h(a, t)$ (green, dashed)

Figure 13: Decomposition of per capita health expenditures and demand

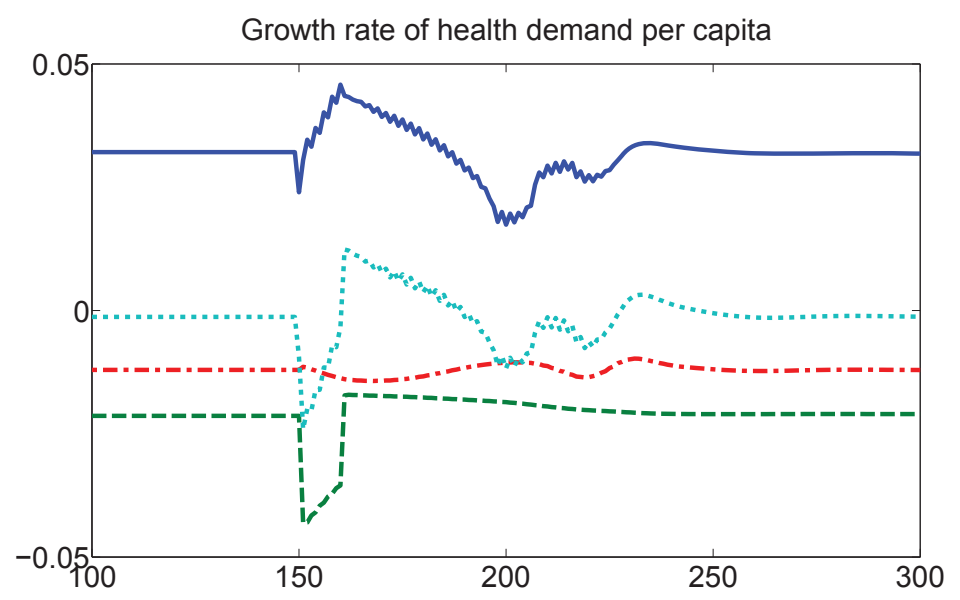

Figure 14: Impact of individual demand change (blue, solid), death-related impact (red, dashed-dotted), birth-related impact (green, dashed) on the growth rate of health demand per capita; Total growth rate is given by the cyan, dotted line.

a more gradual increase in the growth of individual demand levels (blue, solid line), following the ageing of the new-born cohorts as well as the price-driven boom in the demand for health care. Ultimately, this effect is reversed with growth rates of average demand for health care falling below their benchmark. Interestingly, the baby boom does not have any sizeable impact on the growth of the per capita consumption of care through changes in the death rate or the level or composition of health care among the deceased (red, dashed-dotted line).

We can thus conclude the following.

Result 3 (i) An (unanticipated) baby boom leads to a supply-side boom, followed by a demand-side boom in the market for health care. (ii) The two booms translate 
into a twin peak of the Medicare tax rate. (iii) In terms of health expenditure per capita, the supply-side boom is dampened in as far as the price for health care is lowered, whereas the demand-side boom is magnified by a higher price for care. (iii) Cohorts who are retired at the point of the baby boom benefit unambiguously from a level of consumption and health care in excess of the benchmark. (iv) Baby boom cohorts suffer a reduction in consumption and health care below the benchmark when they are old, but by advancing consumption and health care sustain higher levels of the two variables over their middle ages. (v) Per capita demand for health care is subject to strong movements of the average individual demand for health care (for a given age-structure) and of the age structure (for a given age-profile of demand): here the initial (final) dip of per capita expenditure below the baseline is driven by a young age-structure (low level of individual demand), while the peak of per capita expenditure is driven by both an ageing population and a relatively high level of individual demand. (vi) Changes in the per capita consumption of health care, following a baby boom, are predominantly driven by changes in the birth rate and in individual consumption levels, with changes in the death rate playing a negligible role.

\subsection{Discussion}

At this point, we should highlight a number of important, if obvious, caveats to our numerical analysis: (i) As we have argued above, we have deliberately chosen to freeze the benchmark economy to the state of the US economy in 2003. This is to isolate the impact of medical progress and demographic change in an experimental way. This allows us to identify the "pure" effects of these changes, but of course it also implies that these do not reflect the real world time trends. (ii) Some of the modeling choices represent abstractions. For instance, the first two experiments, contrasting the impact of a medical innovation in the absence of any anticipation as opposed to the presence of perfect anticipation are both extremal cases. (iii) We would expect that some of the effects we have identified turn on institutional detail. For instance, saving responses to medical or demographic change crucially depend on the design of the pension system and health insurance. In accordance with the US economy, our specification involves a defined-benefit, pay-as-you-go pension scheme, tax-financed health insurance for the elderly (Medicare), and private insurance based on age-specific premiums for the young and as supplementary insurance for the old. Changing these details, e.g. toward a greater share of publicly financed health care as in many European countries, is clearly 
not only prone to change the benchmark but also some of the experimental impacts. With these caveats in mind, we believe that the experiments reveal a number of salient transmission channels, when it comes to the impact of medical change and population change. The most important of these include:

- the general equilibrium feed-back of price changes on the individual life-cycle allocation, including both level effects and shifts in the age-distribution of the choices. The former are triggered by changes in the wage rate, the latter are triggered by changes in the interest rate, while the price for health care has both level and distributional effects;

- the way in which changes to the level and age-distribution of individual supply and demand combine with changes in the age-distribution of the population to form changes in the aggregate supply and demand;

- the way in which initial shocks evolve over time, inducing a distinct time pattern of macro-economic consequences; and

- the extent to which exogenous shocks are anticipated.

As our numerical examples show, these general mechanisms will need to be carefully accounted for in theoretical and empirical models, analysing the impact of medical change and population change on the supply of and demand for health care.

\section{Conclusion}

We have set out and solved (to the extent possible) an OLG model built around the endogenous demand and supply of health care. In contrast to much of the received macro-economic literature on health and health care, our model involves a rich model of the life-cycle, based on a realistic pattern of mortality. This allows us to characterise in detail the individual life-cycle allocation of consumption and health care, and to construct macro-economic aggregates that are based on a realistic age-structure of the population. At the micro-economic level, we can show in detail how the demand for health care depends on technology, the price for care and, importantly, the willingnessto-pay, as summarised by the value of life.

Solving the macro-economic model, we can derive the aggregate dynamics. In particular, we are able to characterise the dynamics of the aggregate demand for health care, as the age-weighted sum of changes over time in the age-specific individual demand 
for health care and a "generational turn-over" term, defined as the sum of the additional demand generated by the new incoming cohort (births) and the loss in demand due to the deaths across all of the cohorts. The dynamic equation for the change in health care expenditure constitutes an analogon to the better-known aggregate Euler equation for consumption, and it should provide a useful basis for structuring empirical or prognostic work on the evolution of the demand for health care.

Our numerical analysis is designed to provide a quasi-experimental identification of the transmission channels between individual choices and macro-economic dynamics, based on a calibration of the model to the US economy in the year 2003. The knowledge of these transmission channels is important for a good understanding of the macro-economic evolution of the health care sector and its interaction with other parts of the economy. Facing the trade-off between the ability to isolate (in an experimental way) the pure effects of medical and demographic shocks and arriving at a realistic description of the development of economy we have currently come down on the "pure" side. Subject to this caveat, our numerical experiments yield a number of policy relevant, and potentially challenging, insights.

First, about half of the impact on the individual demand for health care of a mortality reducing innovation is absorbed by an increase in the price for medical care. This not withstanding health expenditure per capita increases by some $13 \%$, with 1 percentage point owing to price inflation, 2 percentage points owing to a shift in the age-structure towards older individuals with greater consumption of health care, and 10 percentage points owing to an increase in individual demand.

Second, for an economy with social security and health care organised in similarity to the US (as of 2003), a costless medical innovation does not have a negative impact on economic performance, as measured by GDP. This is despite a shift in economic activity toward health care and a reduction in the employment rate due to a growing population of pensioneers. The main mitigating channel is the accumulation of additional savings/capital for the purpose of financing consumption over an extended life-course and purchasing more effective health care at a higher price. Indeed, this channel is very much in line with evidence for the US on savings related to health expenditures in old age (e.g. De Nardi et al. 2010). Two caveats are worth of note here: The cost of medical innovation, e.g. through the absorption of production factors in an medical R\&D sector may after all induce a drag on economic growth (Jones 2011). In addition, the question as to whether additional savings are induced in the wake of a medical innovation is likely to depend on the particular design of the social security 
system. To the extent that expenditures during retirement are financed through public transfers, the savings response is prone to be weaker, implying that the reduction in the employment rate is not sufficiently offset through the accumulation of capital. Additional offsetting impacts arise if health improvements not only translate into lower mortality but also into a greater propensity to provide labor into older ages (Kuhn and Prettner 2016).

Third, mortality reducing medical innovations tend to come with a reduction in the value of life over large parts of the life-course. This finding has two interesting ramification. At face value, the reduction in the value of life arises from a reallocation by the individual of resources from consumption to health care. While per se, this is reflecting an efficient response by the individual to the availability of more effective health care, it also implies that individuals may be less willing to prevent risks to their life. Thus, some of the benefits of medical innovations in terms of improved survival prospects may well be offset by the adoption of less healthy life-styles. As we have shown the reduction in the value of life also implies a reduction in the effective (quality-adjusted) price of medical care as triggered by the innovation. This is in line with evidence for the US, as provided in Cutler et al. (1998) and suggests that in settings in which individuals choose the demand for health care, the value of life can be interpreted in analogy to the marginal rate of substitution, where a decline is associated with a shift in consumption toward the good in question (in this case health care).

Fourth, anticipation of a medical innovation comes with a reduction in the demand for medical care prior to the innovation with consequences for the sectoral structure and the price structure. In particular, we find that individuals boost their saving in anticipation of the advance, reducing the consumption of health care, amounting to a deferral, but also final goods consumption. The resulting increase in the capital stock per capita is strong enough to trigger a temporary economic boom. The boom is accompanied by a peak in the nominal price for medical care at the point of innovation, leading to a dampening of the impact of medical innovation on the effective price of care. While these effects are only temporary and vanish over the transition to the long-run steady state, they suggest that care needs to be taken about possible anticipation effects when assessing the impacts of medical innovation on economic and health outcomes. While we are unaware of empirical evidence on anticipation effects in the context of medical innovation, their empirical relevance has been established in the context of health policy reform (Hu et al. 2014, Alpert 2015) and strikes us as at 
least conceivable in the innovation context, too.

Fifth, a temporary baby-boom triggers two partially offsetting ripples in the per capita consumption of health care: The shift in age-structure from an initially youngerthan-baseline population to an older-than-baseline population is reflected in an initial reduction in per capita consumption of health care while the boomers are young, followed by a peak, once they have turned old. The age-structure impacts on the demand for health care are partially offset by opposing supply-driven changes in the individual consumption of health care: initially, the low price during the labour boom triggers an increase in individual consumption. This is followed by a slump in individual demand due to price and tax increases once the baby-boomers turn old and enter retirement. The price and income driven changes in individual demand follow the changes in the age-structure with a delay. While the rejuvenation of the population during the early phase of the baby boom leads to a reduction in the per capita demand for health care, both waves reinforce each other during the middle phase, inducing a peak in per capita demand. Eventually, the slump in individual demand is dominating during the final phase of the baby boom. When comparing the life-cycle profiles of different cohorts, it turns out that elderly cohorts who are still alive at the time of the baby boom tend to benefit from both greater consumption and health care, whereas the baby boom cohorts face a reduction of consumption and health care in their old age. This notwithstanding, baby boom cohorts are facing modest increases in both consumption and health care utilisation during their prime age.

We have already hinted at the fact that our model does not yet provide an accurate description of the dynamics of the economy. In a next step we will therefore include more realistic dynamics in regard to productivity growth as well as background trends of medical progress and population. Further work will involve an analysis of health policies, including the derivation of a first-best allocation as a benchmark for assessing the efficiency of the decentralised economy and the need for policy reforms. We also plan to include a medical R\&D sector in order to analyse the joint dynamics within the nexus of health expenditure, ageing and medical progress. 


\section{References}

[1] Aldy JE and WK Viscusi (2008), Adjusting the value of a statistical life for age and cohort effects, Review of Economics and Statistics 90, 573-581.

[2] Alpert A (2015), The anticipatory effects of Medicare Part D on drug utilization. Mimeo.

[3] Baltagi BH, F Moscone and E Tosetti (2012), Medical technology and the production of health care, Empirical Economics 42, 395-411.

[4] Bhattacharya J and X Qiao (2007), Public and private expenditures on health in a growth model, Journal of Economic Dynamics and Control.31, 2519-2535.

[5] Breyer F, J Costa-Font and S Felder (2010), Ageing, health, and health care, Oxford Review of Economic Policy 26, 674-690.

[6] Breyer F and S Felder (2006), Life Expectancy and Health Care Expenditures: A New Calculation for Germany Using the Costs of Dying, Health Policy 75, 178-186.

[7] Breyer F, N Lorenz and T Niebel (2015), Health Care Expenditures and Longevity: Is there a Eubie Blake Effect? European Journal of Health Economics 16, 95-112.

[8] Chakraborty S (2004), Endogenous lifetime and economic growth, Journal of Economic Theory 116, 119-137.

[9] Cremieux P-Y, P Ouellette and C Pilon (1999), Health care spending as determinants of health outcomes, Health Economics 8, 627-639.

[10] Cutler DM (2007), The lifetime costs and benefits of medical technology, Journal of Health Economics 26, 1081-1100.

[11] Cutler DM and RS Huckman (2003), Technological development and medical productivity: the diffusion of angioplasty in New York State, Journal of Health Economics 22, 187-217.

[12] Cutler DM, M McClellan, JP Newhouse and D Rehmer (1998), Are medical prices declining? Evidence from heart attach treatments, Quarterly Journal of Economics 113, 991-1024.

[13] Dalgaard C-J and H Strulik (2014), Optimal Aging and Death: Understanding the Preston Curve, Journal of the European Economic Association 12, 672-701. 
[14] De Nardi M, E French and JB Jones (2010), Why Do the Elderly Save? The Role of Medical Expenses, Journal of Political Economy 118, 39-75.

[15] Donahoe GF (2000), Capital in the national health accounts. Paper submitted to Health Care Financing Administration.

[16] Dormont B, M Grignon and H Huber (2006), Health Expenditure Growth: Reassessing the Threat of Ageing, Health Economics 15, 947-963.

[17] Ehrlich I (2000), Uncertain lifetime, life protection, and the value of life saving, Journal of Health Economics 19, 341-367.

[18] Ehrlich I and H Chuma (1990), A Model of the Demand for Longevity and the Value of Life Extension, Journal of Political Economy 98, 761-782.

[19] European Union (2012), The 2012 Ageing Report: Economic and Budgetary Projections for the EU27 Member States (2010-2060).

[20] Feichtinger G, RF Hartl PM Kort and VM Veliov (2006), Anticipation effcts of technological progress on capital accumulation: a vintage capital approach, Journal of Economic Theory 126, 143-164.

[21] Grossman M (1972), On the Concept of Health Capital and the Demand for Health, Journal of Political Economy 80, 223-255.

[22] Grossmann V and H Strulik (2014), Optimal health and pension policy with biologically founded human ageing. Mimeo.

[23] Johansson P-O (2002), On the definition and age-dependency of the value of a statistical life, Journal of Risk and Uncertainty 25, 251-263.

[24] Jones CI (2011), Life and growth, NBER Working Paper 17094.

[25] Hall RE and Jones CI (2007), The Value of Life and the Rise in Health Spending, Quarterly Journal of Economics 122, 39-72.

[26] Hansen GD and S Imrohoroglu (2008), Consumption over the lifecycle: the role of annuities, Review of Economic Dynamics 11, 566-583.

[27] Heijdra BJ and JO Mierau (2012), The individual life-cycle, annuity market imperfections and economic growth, Journal of Economic Dynamics and Control 36, 876-890. 
[28] Hu T, SL Decker and S-Y Chou (2014), The impact of health insurance expension on physician treatment choice: Medicare Part D and physician prescribing, NBER Working Paper 20708.

[29] Kaplan CM and Y Zhang (2016), Anticipatory behavior in response to Medicare Part D's gap, Health Economics, in press.

[30] Karlsson M and F Klohn (2014), Testing the red-herring hypothesis on an aggregated level: ageing, time-to-death and care costs for older people in Sweden, European Journal of Health Economics 15, 533-551.

[31] Koijen RSJ, TJ Philipson and H Uhlig (2016), Financial health economics, Econometrica 84, 195-242.

[32] Kuhn M and K Prettner (2015), Population structure and consumption growth. Evidence from National Transfer Accounts. Vienna University of Technology, ECON Working Paper 01/2015.

[33] Kuhn M and K Prettner (2016), Growth and welfare effects of health care in knowledge based economies, Journal of Health Economics 46, 100-119.

[34] Kuhn M, S Wrzaczek and J Oeppen (2010), Recognizing progeny in the value of life, Economics Letters 107, 17-21.

[35] Kuhn M, S Wrzaczek, A Prskawetz and G Feichtinger (2011), Externalities in a life cycle model with endogenous survival, Journal of Mathematical Economics 47, 627-641.

[36] Kuhn M, S Wrzaczek, A Prskawetz and G Feichtinger (2015), Optimal choice of health and retirement in a life-cycle model, Journal of Economic Theory 158A, .186-212.

[37] Lichtenberg FR (2004), Sources of U.S. longevity increase, 1960-2001, Quarterly Review of Economics and Finance 44, 369-389.

[38] Ludwig A, T Schelkle and E Vogel (2012), Demographic change, human capital and welfare, Review of Economic Dynamics 15, 94-107.

[39] Martini ME, N Garrett, T Lindquist and GJ Isham (2007), The Boomers Are Coming: A Total Cost of Care Model of the Impact of Population Aging on Health 
Care Costs in the United States by Major Practice Category, Health Services Research 43, 201-218.

[40] Meara E, C White and DM Cutler (2004), Trends in Medical Spending by Age, 1963-2000, Health Affairs 23, 176-183.

[41] Mierau JO and SJ Turnovsky (2014), Capital accumulation and the sources of demographic change, Journal of Population Economics 27, 857-894.

[42] Murphy KM and RH Topel (2006), The Value of Health and Longevity, Journal of Political Economy 114, 871-904.

[43] Reichling F and K Smetters (2015), Optimal annuitization with stochastic mortality and correlated medical costs, American Economic Review 105, 3273-3320.

[44] Ried W (1998), Comparative dynamic analysis of the full Grossman model, Journal of Health Economics 17, 383-425.

[45] Rosen S (1988), The Value of Changes in Life Expectancy, Journal of Risk and Uncertainty 11, 285-304.

[46] Schneider MT and R Winkler (2016), Growth and welfare under endogenous lifetime, University of Bath: Bath Economics Research Papers No. 47/16.

[47] Shang B and D Goldman (2007), Does Age or Life-Expectancy Better Predict Health Care Expenditures?, Health Economics 17, 487-501.

[48] Shepard DS and RJ Zeckhauser (1984), Survival versus consumption, Management Science 30, 423-439.

[49] Veliov VM (2003), Newton's method for problems of optimal control of heterogeneous systems , Optimization Methods and Software 18(6), 689-703.

[50] Warshawsky M (1988), Private annuity markets in the United States: 1919-1984, Journal of Risk and Insurance 55, 515-528.

[51] Yew SL and J Zhang (2014), Longevity, social security, and public health programs in a dynastic model of capital accumulation, health investments, and fertility.

[52] Zhao K (2014), Social security and the rise in health spending, Journal of Monetary Economics 64, 21-37. 
[53] Zweifel P, S Felder and M Meier (1999), Ageing of population and health care expenditure: a red herring? Health Economics 8, 485-496.

[54] Zweifel P, L Steinmann and P Eugster (2005), The Sisyphus Syndrome in Health Revisited, International Journal of Health Care Finance and Economics 5, 127-145. 


\section{$7 \quad$ Appendix}

\subsection{A1: Optimal solution to the individual life-cycle problem}

The individual's life-cycle problem, i.e. the maximisation of (1) subject to (2) and (3) can be expressed by the Hamiltonian

$$
\mathcal{H}=u S-\lambda_{S} \mu S+\lambda_{k}\left(r k+l w-c-\phi p_{H} h-\tau+\pi+d_{H}+s\right),
$$

leading to the first-order conditions

$$
\begin{aligned}
\mathcal{H}_{c} & =u_{c} S-\lambda_{k}=0, \\
\mathcal{H}_{h} & =-\lambda_{S} \mu_{h} S-\lambda_{k} \phi p_{H}=0,
\end{aligned}
$$

and the adjoint equations

$$
\begin{aligned}
& \dot{\lambda}_{S}=(\rho+\mu) \lambda_{S}-u, \\
& \dot{\lambda}_{k}=(\rho-r) \lambda_{k} .
\end{aligned}
$$

Optimality conditions (15) and (16): Evaluating (32) at two different ages/years $(a, t)$ and $(\widehat{a}, t+\widehat{a}-a)$, equating the terms and rearranging gives us

$$
\begin{aligned}
\frac{u_{c}(\widehat{a}, t+\widehat{a}-a)}{u_{c}(a, t)} & =\frac{\lambda_{k}(\widehat{a}, t+\widehat{a}-a)}{\lambda_{k}(a, t)} \frac{S(a, t)}{S(\widehat{a}, t+\widehat{a}-a)} \\
& =\exp \left\{\int_{a}^{\widehat{a}}[\rho-r(t+\widehat{\widehat{a}}-a)] d \widehat{\widehat{a}}\right\} \frac{S(a, t)}{S(\widehat{a}, t+\widehat{a}-a)},
\end{aligned}
$$

which is readily transformed into the Euler equation (15) as given in the main body of the paper.

Inserting (32) into (33) allows to rewrite the first-order condition for health care as

$$
-\mu_{h}(a, t) \frac{\lambda_{S}(a, t)}{u_{c}(\cdot)}=\phi(a, t) p_{H}(t)
$$

Integrating (34) we obtain

$$
\lambda_{S}(a, t)=\int_{a}^{\omega} u(\widehat{a}, t+\widehat{a}-a) \exp \left[-\int_{a}^{\widehat{a}}(\rho+\mu) d \widehat{\widehat{a}}\right] d \widehat{a}
$$


Using this, we can express the private VOL as

$$
\psi(a, t):=\frac{\lambda_{S}(a, t)}{u_{c}(a, t)}=\int_{a}^{\omega} \frac{u_{c}(\widehat{a}, t+\widehat{a}-a)}{u_{c}(a, t)} \frac{u(\widehat{a}, t+\widehat{a}-a)}{u_{c}(\widehat{a}, t+\widehat{a}-a)} \exp \left[-\int_{a}^{\widehat{a}}(\rho+\mu) d \widehat{\widehat{a}}\right] d \widehat{a} .
$$

Substituting from (36) and rearranging we obtain (17) as given in the main body of the paper. Inserting this into (37) gives condition (16) in the main body of the paper.

Dynamics (19) and (20): Total differentiation of (32) with respect to time gives

$$
\begin{aligned}
& u_{c c} S \dot{c}+u_{c} \dot{S}-\dot{\lambda}_{k} \\
= & u_{c c} S \dot{c}-u_{c} \mu S-(\rho-r) \lambda_{k} \\
= & u_{c c} S \dot{c}-(\rho-r+\mu) u_{c} S=0 .
\end{aligned}
$$

From this we obtain the consumption dynamics (19) as given in the main body of the paper.

Total differentiation of $-\mu_{h}(a, t) \psi(a, t)-\phi(a, t) p_{H}(t)=0$ with respect to time gives

$$
-\left(\mu_{h h} \dot{h}+\mu_{h a}++\mu_{h M} \dot{M}\right) \psi-\mu_{h} \dot{\psi}-\phi \dot{p}_{H}-\dot{\phi} p_{H}=0
$$

from which we obtain the dynamics for health care as,

$$
\dot{h}=\frac{-1}{\mu_{h h}}\left(\mu_{h a}+\mu_{h M} \dot{M}+\frac{\phi \dot{p}_{H}+\dot{\phi} p_{H}+\mu_{h} \dot{\psi}}{\psi}\right)
$$

which is easily transformed into (20), as reported in the main body of the paper.

\subsection{A2: Characterisation of general equilibrium}

For each period $t$ we have the following unknown variables:

- inputs $\left\{K_{Y}(t), K_{H}(t), L_{Y}(t), L_{H}(t)\right\}$,

- prices $\left\{r(t), w(t), p_{H}(t)\right\}$,

- aggregate demand $\{C(t), H(t)\}$,

- aggregate net saving, equivalent to the change in the capital stock $K(t)$,

summing up to 10 variables. These are determined through 
- 4 first-order conditions on factor inputs (22)-(25), which give the factor demand functions $\left\{K_{Y}^{d}(r, w ; A, M, B), K_{H}^{d}\left(r, w, p_{H} ; M, B\right)\right.$, $\left.L_{Y}^{d}(r, w ; A, M, B), L_{H}^{d}\left(r, w, p_{H} ; M, B\right)\right\}$, depending on prices as well as on technology and population $\{A, M, B\} ;{ }^{33}$

- a set of first-order conditions (15) and (16) for $a \in[0, \omega]$, which together with the individual's life-cycle budget constraint determine the age-specific levels of consumption $c(a, t)$ and health care $h(a, t)$. Aggregation according to (8) and (9) gives the demand for consumption $C\left(r, w, p_{H} ; M, B, \phi\right)$ and health care $H^{d}\left(p_{H} ; M, B, \phi\right)$, depending on the three prices as well as on technology, population and the vector of co-insurance rates; ${ }^{34}$

- 4 market clearing conditions

$$
\begin{aligned}
K_{Y}^{d}(r, w ; A, M, B)+K_{H}^{d}\left(r, w, p_{H} ; M, B\right) & =K, \\
L_{Y}^{d}(r, w ; A, M, B)+L_{H}^{d}\left(r, w, p_{H} ; M, B\right) & =L(M, B), \\
F\left(M, K_{H}^{d}\left(r, w, p_{H} ; M, B\right), L_{H}^{d}\left(r, w, p_{H} ; M, B\right)\right) & =H^{d}\left(p_{H} ; M, B, \phi\right), \\
\left.Y\left(K_{Y}^{d}(r, w ; A, M, B), A L_{Y}^{d}(r, w ; A, M, B)\right)\right) & =C\left(r, w, p_{H} ; M, B, \phi\right)+\dot{K}+\delta K,
\end{aligned}
$$

which determine the set of equilibrium prices $\left\{r^{*}(A, M, B, \phi, \dot{K})\right.$, $\left.w^{*}(A, M, B, \phi, \dot{K}), p_{H}^{*}(A, M, B, \phi, \dot{K})\right\}$.and aggregate net saving, as captured by $\dot{K}$.

\subsection{A3: Derivation of dynamic system}

The dynamics of the population (27) are immediately given by the time derivative of (6). The dynamics of capital (28) follow both immediately from the goods market equilibrium $Y(t)=C(t)+\delta K(t)+K(t)$ as well as from the aggregate accumulation of wealth. To see this, consider the time derivative of (7), as given by

$$
\dot{K}(t)=\int_{0}^{\omega}[\dot{k}(a, t) N(a, t)+k(a, t) \dot{N}(a, t)] d a+B(t) k(0, t) .
$$

\footnotetext{
${ }^{33}$ Note here that $K_{Y}^{d}(r, w ; A, M)$ and $L_{Y}^{d}(r, w ; A, M)$ may vary with $M$ and $B$ through its impact on the aggregate supply of effective labour $L$.

${ }^{34}$ Through the life-cycle budget constraint and the individual Euler equation the demand function $C(\cdot)$ is also contingent on the expectation about future prices over the remaining life-course. The same applies to the demand function $H^{d}(\cdot)$ for which the future price paths filter in through the VOL.
} 
Assuming that individuals enter without wealth, i.e. $k(0, t)=0$, noting that $N(a, t)=$ $-\bar{\mu}(a, t) N(a, t)$ and inserting from (3) we can write

$$
\begin{aligned}
& \dot{K}(t)=\int_{0}^{\omega}\left\{\begin{array}{c}
{[r(t)-\mu(a, t)] k(a, t)+w(t) l(a)-c(a, t)} \\
-\phi(a, t) p_{H}(t) h(a, t)-\tau(a, t) \\
+\pi(a, t)+d_{H}(t)+s(t)
\end{array}\right\} N(a, t) d a \\
& =r(t) K(t)+w(t) L(t)-C(t)-p_{H}(t) H_{\phi}(t)-\Upsilon(t)+\Pi(t)+d_{H}(t) N(t) \\
& -\int_{0}^{\omega}[\mu(a, t) k(a, t)-s(t)] N(a, t) d a \\
& =r(t) K(t)+w(t) L(t)-C(t)-p_{H}(t) H(t)+d_{H}(t) N(t),
\end{aligned}
$$

where the second line follows under use of the appropriate aggregation and where the third line follows when noting that $\int_{0}^{\omega} \mu(a, t) k(a, t) N(a, t) d a=\Upsilon_{B}(t)=\int_{0}^{\omega} s(t) N(a, t) d a$ by (13) and (14). Profit maximisation in the neoclassical production sector implies $r(t) K_{Y}(t)=Y(t)-w(t) L_{Y}(t)-\delta K_{Y}(t)$, while (11) can be rewritten to $r(t) K_{H}(t)=$ $p_{H}(t) F(t)-w(t) L_{H}(t)-\delta K_{H}(t)-V_{H}(t)$. Adding these, while observing the equilibrium conditions $L_{Y}(t)+L_{H}(t)=L(t)$ and $K_{Y}(t)+K_{H}(t)=K(t)$ gives $r(t) K(t)=$ $Y(t)-w(t) L(t)-\delta K(t)+p_{H}(t) F(t)-V_{H}(t)$. Substituting this into (38), while observing $F(t)=H(t)$ and $V_{H}(t)=d_{H}(t) N(t)$, we obtain the expression in (28).

Differentiation of (8) with respect to time gives

$$
\dot{C}(t)=\int_{0}^{\omega}[\dot{c}(a, t) N(a, t)+c(a, t) \dot{N}(a, t)] d a+B(t) c(0, t) .
$$

Substituting from (19) and noting that $N(a, t)=-\mu(a, t) N(a, t)$ we obtain (29). Differentiation of (9) with respect to time gives

$$
\dot{H}(t)=\int_{0}^{\omega}[\dot{h}(a, t) N(a, t)+h(a, t) \dot{N}(a, t)] d a+B(t) h(0, t) .
$$

Noting that $N(a, t)=-\mu(a, t) N(a, t)$ we obtain $(30)$.

\subsection{A4: Solving the numerical problem}

We pursue the following steps towards tracing out the numerical solution, sketched here for the benchmark scenario, using the specific functional forms presented in section 5 :

1. We derive from the first-order condition for consumption (15) the relationship 


$$
\left[c\left(a, t_{0}+a\right)-c_{0}\right]^{-\sigma}=\left[c\left(0, t_{0}\right)-c_{0}\right]^{-\sigma} \exp \left\{\int_{0}^{a}\left[\rho-r\left(t_{0}+\hat{a}\right)+\mu(\hat{a})\right] d \hat{a}\right\} .
$$

2. We derive the life-cycle budget constraint

$$
\int_{0}^{\omega}\left[\begin{array}{c}
w\left(t_{0}+a\right) l(a)-c\left(a, t_{0}+a\right)+\pi(a, t) \\
-\phi(a, t) p_{H}\left(t_{0}+a\right) h\left(a, t_{0}+a\right)-\tau(a, t)+s\left(t_{0}+a\right)
\end{array}\right] R(a, 0) d a=0,
$$

with $R(a, 0)$ as given by (18). We then insert (39) and obtain the consumption level

$c\left(0, t_{0}\right)-c_{0}=\frac{\int_{0}^{\omega}\left[\begin{array}{c}w\left(t_{0}+a\right) l(a)-c_{0}+\pi(a, t) \\ -\phi(a, t) p_{H}\left(t_{0}+a\right) h\left(a, t_{0}+a\right)-\tau(a, t)+s\left(t_{0}+a\right)\end{array}\right] R(a, 0) d a}{\int_{0}^{\omega} \exp \left\{\int_{0}^{a}\left[\frac{1-\sigma}{\sigma} r\left(t_{0}+\hat{a}\right)-\frac{\rho+\mu(\hat{a})}{\sigma}\right] d \hat{a}\right\} d a}$

for an individual born at $t_{0}$, contingent on the stream of health care, $h\left(a, t_{0}+a\right)$, and the set of prices $\left\{w\left(t_{0}+a\right), r\left(t_{0}+a\right), p_{H}\left(t_{0}+a\right)\right\}$ over the interval $\left[t_{0}, t_{0}+\omega\right]$.

3. We derive from the first-order condition for health care (16) a vector of agespecific demand levels

$$
h\left(a, t_{0}+a\right)=\left(\frac{\lambda_{s}\left(a, t_{0}+a\right)\left[c\left(a, t_{0}+a\right)-c_{0}\right]^{\sigma} \tilde{\mu}(a) \eta(a) \epsilon(a) M\left(t_{0}+a\right)^{\epsilon(a)}}{\phi(a, t) p_{H}\left(t_{0}+a\right)}\right)^{\frac{1}{1-\epsilon(a)}}
$$

for all $a \in[0, \omega]$.

4. We show in Appendix A5 that the set of prices $\left\{w\left(t_{0}+a\right), p_{H}\left(t_{0}+a\right)\right\}$ as well as all input and output quantities can be expressed in terms of the interest rate $r\left(t_{0}+a\right)$ alone.

5. Using (39) together with (41) we can calculate the life-cycle allocation for consumption, $c\left(a, t_{0}+a\right)$, depending on the allocation for health expenditures, $h\left(a, t_{0}+\right.$ $a), \forall a \in[0, \omega]$ and on the set of prices $\left\{w\left(t_{0}+a\right), r\left(t_{0}+a\right), p_{H}\left(t_{0}+a\right)\right\}$ over the interval $\left[t_{0}, t_{0}+\omega\right]$. Vice versa, the allocation of health expenditures can be calculated from the allocation of consumption and the macroeconomic prices. 
6. We apply these calculations on initial guesses of $c$ and $h$ iteratively. We then use the results as an initial guess to the age-structured optimal control algorithm, as presented in Veliov (2003). This yields an optimal allocation of individual consumption and health expenditures contingent on an initially assumed $r\left(t_{0}+a\right)$.

7. Drawing on this, we apply the following recursive approximation algorithm: (i) Guess an initial interest rate $r\left(t_{0}+a\right)$ and derive the optimal life-cycle allocation. (ii) Based on this, calculate the market interest rate $r^{*}\left(t_{0}+a\right)$ from the capital market equilibrium $K^{d}\left(r\left(t_{0}+a\right), \widehat{w}\left(r\left(t_{0}+a\right)\right)\right)=K^{s}\left(r\left(t_{0}+a\right)\right)$. (iii) Adjust the initial interest rate, so that it approaches $r^{*}\left(t_{0}+a\right)$, e.g. by setting $r_{1}\left(t_{0}+a\right):=$ $r_{0}\left(t_{0}+a\right)+\epsilon\left(r^{*}\left(t_{0}+a\right)-r_{0}\left(t_{0}+a\right)\right), \quad \epsilon \in(0,1]$. The process converges to an interest rate for which households optimise and capital demand equals capital supply. The output market clearing condition, $Y\left(t_{0}+a\right)=C\left(t_{0}+a\right)+\dot{K}\left(t_{0}+\right.$ $a)+\delta K\left(t_{0}+a\right)$ then determines the dynamics of the capital stock to the next period. (iv) This process is reiterated in a recursive way, employing a solution algorithm based on Newton's method. Equations (39)-(41) allow us to verify expost an optimum life-cycle allocation for the focal cohort born at $t_{0}$. While the numerical algorithm cannot determine in a precise way the optimal allocation for other cohorts, it nevertheless structures the allocation in a way that approximates the optimum for all cohorts.

\subsection{A5: Equilibrium relationships with Cobb-Douglas technologies}

Consider the Cobb-Douglas-specifications

$$
\begin{aligned}
& Y(t)=K_{Y}(t)^{\alpha}\left[A(t) L_{Y}(t)\right]^{1-\alpha} \\
& F(t)=M(t)^{\gamma} K_{H}(t)^{\beta}\left[L_{H}(t)\right]^{1-\beta},
\end{aligned}
$$

with $\alpha, \beta \in[0,1]$ and $\gamma \in[-1,1]$. Note that this specification allows for a situation, where for $\gamma<0$ medical progress lowers the production efficiency of health care. This may refer to those cases, where new and resource intensive technologies are employed in order to lower mortality $\mu_{M}<0$.

From the first-order conditions (22), (23), (24) and (25) we then obtain the factor 
demand functions

$$
\begin{aligned}
K_{Y}^{d}(t) & =\frac{\alpha Y(t)}{r(t)+\delta}, \\
L_{Y}^{d}(t) & =\frac{(1-\alpha) Y(t)}{w(t)}, \\
K_{H}^{d}(t) & =\frac{\beta p_{H}(t) F(t)}{r(t)+\delta}, \\
L_{H}^{d}(t) & =\frac{(1-\beta) p_{H}(t) F(t)}{w(t)} .
\end{aligned}
$$

Combining (44) with (45) and (46) with (47) we obtain the equilibrium capital intensity

$$
\begin{aligned}
& k_{Y}^{*}(t):=\quad \frac{K_{Y}^{d}(t)}{L_{Y}^{d}(t)}=\frac{\alpha}{1-\alpha} \frac{w(t)}{r(t)+\delta}, \\
& k_{H}^{*}(t):=\quad \frac{K_{H}^{d}(t)}{L_{H}^{d}(t)}=\frac{\beta}{1-\beta} \frac{w(t)}{r(t)+\delta} .
\end{aligned}
$$

and, thus, $K_{Y}^{d}(t)=k_{Y}^{*}(t) L_{Y}^{d}(t)$. Using $k_{Y}^{*}(t)$ in (42) to rewrite $Y(t)=L_{Y}^{d}(t) A(t)^{1-\alpha}\left(k_{Y}^{*}\right)^{\alpha}$ and inserting this in (45) we can solve for the equilibrium wage as a function of the interest rate

$$
w^{*}(t)=\widehat{w}(r(t) ; A(t))=(1-\alpha) A(t)\left[\frac{\alpha}{r(t)+\delta}\right]^{\frac{\alpha}{1-\alpha}} .
$$

This, in turn, determines the capital intensities $k_{Y}^{*}(t)=\widehat{k}_{Y}(r(t) ; A(t))$ and $k_{H}^{*}(t)=$ $\widehat{k}_{H}(r(t) ; A(t))$. Using the market clearing condition $F\left(p_{H}^{*}(t) ; M(t), K_{H}^{*}(t), L_{H}^{*}(t)\right)=$ $H^{d}\left(p_{H}^{*}(t) ; M(t), B(t)\right)$ and (46) and (47) we obtain the general equilibrium price for health care as

$$
\begin{aligned}
p_{H}^{*}(t) & =\widehat{p}_{H}\left(r(t), w^{*}(t), H_{d}^{*}(t) ; M(t)\right) \\
& =\widehat{p}_{H}(r(t) ; A(t), M(t), B(t)) \\
& =\frac{1}{M(t)^{\gamma}} \frac{(r+\delta)^{\beta} w^{1-\beta}}{\beta^{\beta}(1-\beta)^{1-\beta}} .
\end{aligned}
$$

Reinserting this, we obtain the equilbrium utilisation of health care, as $H^{d}\left(p_{H}^{*}(t) ; M(t), B(t)\right)=\widehat{H}(r(t) ; A(t), M(t), B(t))$. Using (47) we can determine now $L_{H}^{*}(t)=\widehat{L}_{H}\left(p_{H}^{*}(t), w^{*}(t), H_{d}^{*}(t)\right)=\widehat{L}_{H}(r(t) ; A(t), M(t), B(t))$. The labour 
market equilibrium then determines

$$
L_{Y}^{*}(t)=L(t)-L_{H}^{*}(t),
$$

where $L(t)=\widehat{L}(r(t) ; A(t), M(t), B(t)) .{ }^{35}$ This implies the restriction

$$
\widehat{L}(r(t) ; A(t), M(t), B(t)) \geq \widehat{L}_{H}(r(t) ; A(t), M(t), B(t)) .
$$

Given this is satisfied, we now have all inputs and outputs as functions of $r(t)$ and the states $\{A(t), M(t), B(t)\}$.

\footnotetext{
${ }^{35}$ Note that through the impact of the demand for health care on the pattern of survival, labour supply becomes a function of the prices and the states of the economy.
} 


\section{VIENNA INSTITUTE OF DEMOGRAPHY}

\section{Working Papers}

Abel, Guy J., Estimates of Global Bilateral Migration Flows by Gender between 1960 and 2015, VID Working Paper 2/2016.

Testa, Maria Rita, Valeria Bordone, Beata Osiewalska and Vegard Skirbekk, The Relation between Mother's Socio-Economic Status and Daughter's Fertility Intentions in Austria, Italy, Bulgaria, and Norway, VID Working Paper 1/2016.

Hoffmann, Roman and Raya Muttarak, A Tale of Disaster Experience in Two Countries: Does Education Promote Disaster Preparedness in the Philippines and Thailand, VID Working Paper 9/2015.

Klotz, Johannes and Richard Gisser, Mortality Differentials by Religious Denomination in Vienna 1981-2002, VID Working Paper 8/2015.

Steiber, Nadia and Barbara Haas, Overworked or Underemployed? Actual and Preferred Household Employment Patterns in the Context of the Economic Crisis, VID Working Paper 7/2015.

Beaujouan, Eva, Zuzanna Brzozowska and Krystof Zeman, Childlessness Trends in Twentieth-Century Europe: Limited Link to Growing Educational Attainment, VID Working Paper 6/2015.

Abel, Guy, Estimates of Global Bilateral Migration Flows by Gender between 1960 and 2010, VID Working Paper 5/2015.

Spijker, Jeroen, Alternative Indicators of Population Ageing: An Inventory, VID Working Paper 4/2015.

Kuhn, Michael and Klaus Prettner, Population Structure and Consumption Growth: Evidence from National Transfer Accounts, VID Working Paper 3/2015.

Sobotka, Tomáš, Low Fertility in Austria and the Czech Republic: Gradual Policy Adjustments, VID Working Paper 2/2015.

Buber-Ennser, Isabella, Aspects of Gender Mainstreaming of Family and Work in Austria, VID Working Paper 1/2015.

Buber-Ennser, Isabella and Ralina Panova, Attitudes towards Parental Employment across Europe, in Australia and in Japan, VID Working Paper 5/2014.

The Vienna Institute of Demography Working Paper Series receives only limited review. Views or opinions expressed herein are entirely those of the authors. 